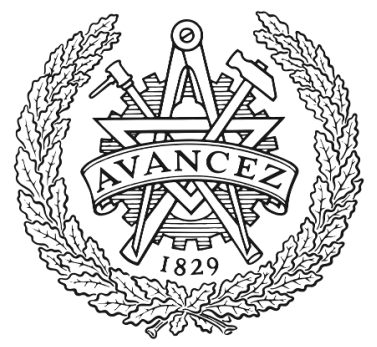

CHALMERS

UNIVERSITY OF TECHNOLOGY

\title{
Accounting for lattice coherency in a two-phase elastic-plastic self-consistent model for nickel-based superalloys
}

Downloaded from: https://research.chalmers.se, 2023-04-26 10:43 UTC

Citation for the original published paper (version of record):

Li, H., Ekh, M., Hörnqvist Colliander, M. et al (2018). Accounting for lattice coherency in a two-phase elastic-plastic self-consistent model for nickel-based superalloys. International Journal of Plasticity, 110: 248-271.

http://dx.doi.org/10.1016/j.ijplas.2018.07.006

N.B. When citing this work, cite the original published paper. 


\title{
Accounting for lattice coherency in a two-phase elastic-plastic self-consistent model for nickel-based superalloys
}

\author{
Hongjia Li $i^{\mathrm{a}, \mathrm{b}, \mathrm{c}, *}$, Magnus Ekh ${ }^{\mathrm{b}}$, Magnus Hörnqvist Colliander ${ }^{\mathrm{a}}$, Fredrik Larsson ${ }^{\mathrm{b}}$ \\ ${ }^{a}$ Department of Physics, Chalmers University of Technology, SE-41296 Göteborg, Sweden \\ ${ }^{b}$ Department of Industrial and Materials Science, Chalmers University of Technology, SE-41296 Göteborg, \\ Sweden \\ ${ }^{c}$ Institute of Nuclear Physics and Chemistry, China Academy of Engineering Physics, Mianyang 621999, \\ People's Republic of China
}

\begin{abstract}
A 2-site elastic-plastic self-consistent (EPSC) model is developed and implemented in order to account for crystallographic texture development and grain morphology evolution under strong correlations between neighbor grains of different phases, both in space and orientation. Predictions of the model adequately fit the published in situ neutron diffraction data for nickel-based superalloys at ambient and elevated temperatures, in which $\gamma$ and $\gamma^{\prime}$ phases exhibit exact cube-cube orientation relationship. Comparison with 2-site model (small strain algorithm, non-rotation scheme) and 1-site model (finite strain algorithm, co-rotation scheme) has been made, and the result shows that the present 2-site model (finite strain algorithm, rotation scheme) leads to better predictions in lattice strain evolution where both rotation of crystal lattice and correlation between inclusions are accounted for, especially when the applied strain is larger than 0.02 for transverse direction and $0.05 \sim 0.18$ for axial direction for the materials studied in this work. Based on a systematic study on the effects of grain-grain interaction and total grain number on the homogenized results, we found that transverse lattice strains of $\gamma(200)$ and/or $\gamma^{\prime}$ (100) are sensitive to the interplay between $\gamma-\gamma^{\prime}$ interaction and evolution of grain orientation distribution with deformation, while that of $\gamma(220)$ and $\gamma^{\prime}$ (110) are sensitive to the initial crystallographic texture.
\end{abstract}

Keywords: Two-phase polycrystal, Nickel-based superalloy, In situ neutron diffraction, 2-Site elastic-plastic self-consistent model, Finite strain

\section{Introduction}

In situ neutron diffraction is used to quantify the evolution of lattice structure within the bulk of many polycrystalline metals. It has provided the foundation of numerous research on polycrystals with various crystalline structures [1 8 ] and phase components [9-15]. These in situ deformation studies have revealed the heterogeneous stress and strain accommodation within polycrystalline aggregates. Due to the reason that individual diffraction peaks are contributed from subsets of grains, rather than individual grains, modeling is frequently required to quantitatively interpret the changes of lattice strains and deformation mechanisms. Self-consistent (SC) modeling has been the focus of attention as a means of interpreting the

\footnotetext{
*Corresponding author. Tel. +46 317726773

Email address: hongjia.li@chalmers.se (Hongjia Li)
} 
experimental data using simulations of the mechanical response of aggregate in terms of that of individual crystals [1-3, 5-8, 10, 12 14, 16].

Most of the SC models are developed based on the Hill SC approach [17], which was first implemented by Hutchinson [18]. In the model, it assumes that each grain is an ellipsoidal inclusion embedded in an infinite Homogeneous Equivalent Medium (HEM), the properties of which are the average response of all grains in the aggregate. The mechanical response of each grain can be obtained using Eshebly's solution [19]. Recent efforts on development and improvement of SC models have been motivated by the need of capturing elastic-plastic deformation behaviors of individual phases in two-phase alloys. For two-phase polycrystals, there is a strong correlation in morphology and / or orientation between neighbor grains due to the vicinity effect between two phases. One way to account for the effect of this correlation is to accurately define the localized hardening by introducing a thin reinforcement layer between the hard phase and the HEM (i.e. coated ellipsoidal inclusion scheme [20]) for the case where the two phases do not have orientation relationship [21] or incorporating the effect of anti-phase boundary on the initial resistance to precipitate shearing for the case where the two phases have orientation relationship [22] based on the 1-site SC algorithm [23, 24]. But these models are mostly focused on the overall mechanical response under cyclic loading, including stress-strain curve and texture. They have not been applied to simulate lattice strains to interpret deformation behaviors of individual phases. Another way is to consider two interacting grains of different phases deforming in the HEM [25] (i.e. 2-site SC modeling). Typical examples of two-phase materials that have an orientation relationship between phases include the lamellar $(\alpha+\beta)$ titanium (Ti) alloys and nickel-based (Nibased) superalloys. The crystal lattices of the two phases of these alloys will co-rotate since they share slip systems under plastic deformation. Two Ti alloys exhibiting different microstructures were studied by Lebensohn and Canova [25] using a 2-site large strain viscoplastic self-consistent (VPSC) model. Influences of grain shape and correlation between phases on texture formation were found and well discussed. However, VPSC models do not include elastic deformation and hence cannot be used to study lattice strains. Daymond et al. 10] have developed a 2-site elastic-plastic self-consistent (EPSC) model to study lattice strain evolution of Ni-based superalloys with various $\gamma^{\prime}$ microstructures at ambient and elevated temperatures [10, 12, 13]. In the model, uniform effective eigen-strain is used for the 2-site problem to make the ellipsoidal pair deform as a unit within the HEM. Moreover, small total deformations are assumed and no lattice rotation or texture development is incorporated. The model is in good agreement with experimental lattice strains parallel to the tensile stress axis (axial lattice strains), but has only qualitative agreement with that perpendicular to the tensile stress axis (transverse lattice strains) for most of the cases. A lack of quantitative agreement between predicted transverse lattice strains and those measured experimentally remains to be elucidated.

The first objective of the present study is to develop and implement a 2-site EPSC model for finite strain applications by accounting for crystallographic texture development and grain morphology evolution under strong correlations between the two interacting grains of different phases. The second objective is to test the predictive capabilities of the model by comparing the predicted results with published in situ neutron diffraction data of $\mathrm{Ni}$ based superalloys. We focus on accurately modeling lattice strain evolution along both axial direction (AD) and transverse direction (TD), which is a sensitive indicator of plastic deformation mechanisms at microscopic levels. The third objective is to compare the results predicted by the 2-site model (finite strain algorithm, rotation scheme), 2-site model (small 
strain algorithm, non-rotation scheme), and 1-site model (finite strain algorithm, co-rotation scheme) to figure out the differences between them. The fourth objective is to investigate the effects of grain-grain interaction on lattice strain evolution, especially that along TD. In Section 2, we describe the formulation and implementation of the EPSC model. Section 3 shows the results of application to superalloys, comparison between models, and the pertinent effects of $\gamma-\gamma^{\prime}$ interaction on the predicted results. Section 4 gives improved fitting results of transverse lattice strains for the previous studies in Section 3 along with detailed discussions on the main factors that contribute to the improvement.

\section{Modeling}

In the single phase EPSC model [17-19, 24, 26, 27], the effect of neighborhood on the mechanical response of each grain is accounted for through the interaction between the grain and the surrounding HEM. As a consequence, the correlation between neighbor grains due to the vicinity effect is completely disregarded. It is a reasonable assumption and works well for single phase materials [1 3, 5-7] and two-phase alloys that do not have orientation relationship between phases [14, 28]. However, the correlation in morphology and orientation between grains of different phases is strong for lattice-coherent two-phase materials, which cannot be neglected. The 2-site SC model [10, 25] considers the interaction of two ellipsoidal inclusions embedded in a HEM. The deformation behavior of each grain is influenced by both the interactions with a global HEM and its local nearest-neighbor grain. In order to model this, we have made a 2-site modification on Neil et al.'s [24] finite strain 1site EPSC implementation. The approach employed in the model is similar to the one used by Lebensohn and Canova 25] for the large strain 2-site VPSC model. Considering boundary constraints between grains within each ellipsoidal pair, extra schemes are applied to guarantee them to co-rotate [29] and deform compatibly.

In what follows, variables with subscript designations are for grain-level quantities, and those without subscripts are for the overall quantities.

\subsection{Formulation}

\subsubsection{2-site EPSC model}

The microstructure of Ni-based superalloys consist of large $\gamma$ grains with a high number density of small coherent $\gamma^{\prime}$ precipitates in the interiors. In the 2-site EPSC model, however, this is represented by two ellipsoidal inclusions, weighted by the respective volume fractions. We consider $N$ such pairs of inclusions which statistically represent the polycrystalline structure of the material. Each pair is embedded in a homogeneous infinite body. The SC model is defined by letting the tangent stiffness of embedding body represent the (weighted) average of that of the modeled inclusions. According to Eshelby's equality [19], the stress rate $\dot{\boldsymbol{\sigma}}_{c}$ and the strain rate $\dot{\boldsymbol{\epsilon}}_{c}$ of the ellipsoidal inclusions in a HEM (or an infinite body) are equal for:

$$
\begin{aligned}
& \dot{\boldsymbol{\sigma}}_{c}=\mathbf{L}_{c}: \dot{\boldsymbol{\epsilon}}_{c} \\
& \dot{\boldsymbol{\sigma}}_{c}=\mathbf{L}:\left(\dot{\boldsymbol{\epsilon}}_{c}-\dot{\boldsymbol{\epsilon}}_{c}^{*}\right)
\end{aligned}
$$

where $c=1,2$ for the two interacting ellipsoidal inclusions (\# 1 and \# 2), respectively. Eq.(17) pertains to an inclusion in a heterogeneous body without eigen-strains, and Eq.(1p) is established for the equivalent situation in a homogeneous body (stiffness $\mathbf{L}$ ) with eigenstrains $\boldsymbol{\epsilon}_{c}^{*}$. 
The eigen-strain rate can be solved for:

$$
\dot{\boldsymbol{\epsilon}}_{c}^{*}=\dot{\boldsymbol{\epsilon}}_{c}-\mathbf{L}^{-1}: \mathbf{L}_{c}: \dot{\boldsymbol{\epsilon}}_{c}=\left(\mathbf{I}-\mathbf{L}^{-1}: \mathbf{L}_{c}\right): \dot{\boldsymbol{\epsilon}}_{c}=\mathbf{A}_{c}^{*}: \dot{\boldsymbol{\epsilon}}_{c}
$$

Here, $\mathbf{A}_{c}^{*}=\mathbf{I}-\mathbf{L}^{-1}: \mathbf{L}_{c}$ is defined for $c=1,2$.

The strain rate deviations $\dot{\boldsymbol{\epsilon}}_{c}-\dot{\boldsymbol{\epsilon}}$ and eigen-strain rate $\dot{\boldsymbol{\epsilon}}_{c}^{*}$ are related according to the following extended Eshelby relations by the four symmetric Eshelby tensors [19, 25, 30]:

$$
\begin{aligned}
& \dot{\boldsymbol{\epsilon}}_{1}-\dot{\boldsymbol{\epsilon}}=\mathbf{P}_{11}: \dot{\boldsymbol{\epsilon}}_{1}^{*}+\mathbf{P}_{12}: \dot{\boldsymbol{\epsilon}}_{2}^{*} \\
& \dot{\boldsymbol{\epsilon}}_{2}-\dot{\boldsymbol{\epsilon}}=\mathbf{P}_{21}: \dot{\boldsymbol{\epsilon}}_{1}^{*}+\mathbf{P}_{22}: \dot{\boldsymbol{\epsilon}}_{2}^{*}
\end{aligned}
$$

where $\mathbf{P}_{11}$ and $\mathbf{P}_{22}$ are ordinary Eshelby tensors for inclusion \# 1 and \# 2, respectively; and $\mathbf{P}_{12}$ and $\mathbf{P}_{21}$ are the coupling factors between the eigen-strain rate of one inclusion and the local deviation in strain rate of the other. Related derivations and expressions for the 2-site Eshelby tensors refer to Berveiller et al. 31] and Lebensohn and Canova [25].

By combining Eqs. (2) and (3) we obtain:

$$
\begin{aligned}
& \dot{\boldsymbol{\epsilon}}_{1}-\dot{\boldsymbol{\epsilon}}=\mathbf{P}_{11}: \mathbf{A}_{1}^{*}: \dot{\boldsymbol{\epsilon}}_{1}+\mathbf{P}_{12}: \mathbf{A}_{2}^{*}: \dot{\boldsymbol{\epsilon}}_{2} \\
& \dot{\boldsymbol{\epsilon}}_{2}-\dot{\boldsymbol{\epsilon}}=\mathbf{P}_{21}: \mathbf{A}_{1}^{*}: \dot{\boldsymbol{\epsilon}}_{1}+\mathbf{P}_{22}: \mathbf{A}_{2}^{*}: \dot{\boldsymbol{\epsilon}}_{2}
\end{aligned}
$$

By some manipulations, we can obtain:

$$
\begin{aligned}
& \dot{\boldsymbol{\epsilon}}_{1}=\mathbf{A}_{1}: \dot{\boldsymbol{\epsilon}} \\
& \dot{\boldsymbol{\epsilon}}_{2}=\mathbf{A}_{2}: \dot{\boldsymbol{\epsilon}}
\end{aligned}
$$

where the concentration tensors $\mathbf{A}_{1}$ and $\mathbf{A}_{2}$ can be found from Eq. (4) as:

$$
\begin{aligned}
& \mathbf{A}_{1}=\left(\mathbf{B}_{2}: \mathbf{C}_{1}^{-1}: \mathbf{B}_{1}-\mathbf{C}_{2}\right)^{-1}:\left(\mathbf{B}_{2}: \mathbf{C}_{1}^{-1}+\mathbf{I}\right) \\
& \mathbf{A}_{2}=\left(\mathbf{B}_{1}: \mathbf{C}_{2}^{-1}: \mathbf{B}_{2}-\mathbf{C}_{1}\right)^{-1}:\left(\mathbf{B}_{1}: \mathbf{C}_{2}^{-1}+\mathbf{I}\right)
\end{aligned}
$$

with

$$
\begin{aligned}
& \mathbf{B}_{1}=\mathbf{I}-\mathbf{P}_{11}: \mathbf{A}_{1}^{*} \\
& \mathbf{C}_{1}=\mathbf{P}_{12}: \mathbf{A}_{2}^{*}
\end{aligned}
$$

and where $\mathbf{B}_{2}$ and $\mathbf{C}_{2}$ can be obtained interchanging indexes 1 and 2 in Eq.(7).

At the macroscopic scale, the corresponding relation between the stress rate and strain rate is:

$$
\dot{\boldsymbol{\sigma}}=\mathbf{L}: \dot{\boldsymbol{\epsilon}}
$$

The relation between macroscopic and microscopic stress rate takes the form [18]:

$$
\dot{\boldsymbol{\sigma}}=\left\langle\dot{\boldsymbol{\sigma}}_{\mathrm{c}}\right\rangle \quad \text { with }\langle\bullet\rangle=\left.\frac{1}{N} \sum_{p=1}^{N} \sum_{c} w_{c} \bullet\right|_{c, p}
$$

where we introduced the weighted average over both populations of inclusions denoted as $\langle\bullet\rangle . N$ is the total number of pairs, and $w_{c}$ is the weight of each inclusion determined by the volume fraction of each phase with $c=1,2$ for phase \# 1 and \# 2, respectively.

After some algebraic calculations on Eqs.(14), (5), (8), and (9), we can obtain the overall SC stiffness:

$$
\mathbf{L}=\left\langle\mathbf{L}_{c}: \mathbf{A}_{c}\right\rangle \quad(c=1,2)
$$

Derivations and expressions of the strain rates of an individual inclusion in both phases used in the implementation are provided in Appendix A. 


\subsubsection{Texture evolution}

In the 2-site small strain approach [10], rotations are ignored. In the finite strain formulations that follow, rotations of the sample, ellipsoid, and crystal lattice are accounted for [29].

(1) Applied macroscopic rotation rate:

$$
\dot{\Omega}=\frac{1}{2}\left(\boldsymbol{l}-\boldsymbol{l}^{\mathrm{T}}\right)
$$

i.e., the anti-symmetric component of the macroscopic velocity gradient $\boldsymbol{l}$.

(2) Local rotation rate:

$$
\dot{\boldsymbol{\omega}}_{c}=\dot{\Omega}+\tilde{\dot{\omega}}_{c}
$$

where $c=1,2$, and the expressions for the deviations from the macroscopic rotation rate $\tilde{\dot{\boldsymbol{\omega}}}_{1}$ and $\tilde{\dot{\omega}}_{2}$ are given in terms of Eshelby tensors in Appendix B.

(3) Crystal lattice rotation rate:

$$
\dot{\boldsymbol{\omega}}_{c}^{\text {lat }}=\dot{\boldsymbol{\omega}}_{c}-\dot{\boldsymbol{\omega}}_{c}^{\mathrm{pl}}
$$

where $\dot{\boldsymbol{\omega}}_{c}^{\mathrm{pl}}$ is the plastic rotation rate, which takes the form:

$$
\dot{\boldsymbol{\omega}}_{c}^{\mathrm{pl}}=\sum_{s} \boldsymbol{q}_{c}^{s} \dot{\gamma}_{c}^{s}
$$

where $c=1,2, \boldsymbol{q}_{c}^{s}=\operatorname{skw}\left(\boldsymbol{b}_{c}^{s} \otimes \boldsymbol{n}_{c}^{s}\right)$ is the skew-symmetric Schmid tensor defined in terms of the slip direction $\left(\boldsymbol{b}_{c}^{s}\right)$ and slip-plane normal $\left(\boldsymbol{n}_{c}^{s}\right)$, and $\dot{\gamma}_{c}^{s}$ is the shear rate on the $s$-th slip system.

For the 2-site model, properly dealing with rotations of ellipsoid and crystal lattice is predominant to accurately describe the correlation in morphology and orientation between the ellipsoidal inclusions within each pair. Reference and local coordinate systems, and schematic 2-D representation of the model are shown in Fig. 1. Morphological and crystallographic orientations of the two inclusions within a pair are the same, but they are different and random among pairs. Ellipsoid \# 2 is randomly placed with respect to ellipsoid \# 1 , which is represented by a vector from the center of ellipsoid \# 1 to that of ellipsoid \# 2 denoted as $\boldsymbol{D}_{12}^{i}$ with $i$ standing for pair number and $\boldsymbol{D}_{12}^{i}=-\boldsymbol{D}_{21}^{i}$ holds. $\boldsymbol{D}_{12}^{i}$ is also different and random among pairs. Weighted average local rotation rate is used to represent the average trend of both sites:

$$
\dot{\boldsymbol{\omega}}_{\mathrm{ave}}=w_{1} \dot{\boldsymbol{\omega}}_{1}+w_{2} \dot{\boldsymbol{\omega}}_{2}
$$

where $w_{1}$ and $w_{2}$ are the weights of each ellipsoid determined by the volume fractions of each phase. As a consequence, the crystal lattice rotation rate calculated via Eq. (13) is changed into the following form:

$$
\dot{\boldsymbol{\omega}}_{c}^{\text {lat }}=\dot{\boldsymbol{\omega}}_{\text {ave }}-\dot{\boldsymbol{\omega}}_{c}^{\mathrm{pl}}
$$

Taking deformation mechanism into account, e.g. shear across $\gamma / \gamma^{\prime}$ boundaries for Alloy RR1000 with fine $\gamma^{\prime}$ microstructure and looping around $\gamma^{\prime}$ particles for the medium and coarse $\gamma^{\prime}$ microstructures [12, 13], two ideal rotation schemes are provided in our implementation, including local co-rotation (i.e. ellipsoid co-rotates while crystal lattice rotates freely for the two inclusions within each pair) and co-rotation (i.e. both ellipsoid and crystal lattice co-rotate). Eq.(16) is used for the local-co-rotation scheme. For the co-rotation scheme, crystal lattice co-rotation rate is defined as:

$$
\dot{\boldsymbol{\omega}}_{\mathrm{corot}}^{\text {lat }}=\frac{1}{2}\left(\dot{\boldsymbol{\omega}}_{1}^{\text {lat }}+\dot{\boldsymbol{\omega}}_{2}^{\text {lat }}\right)
$$




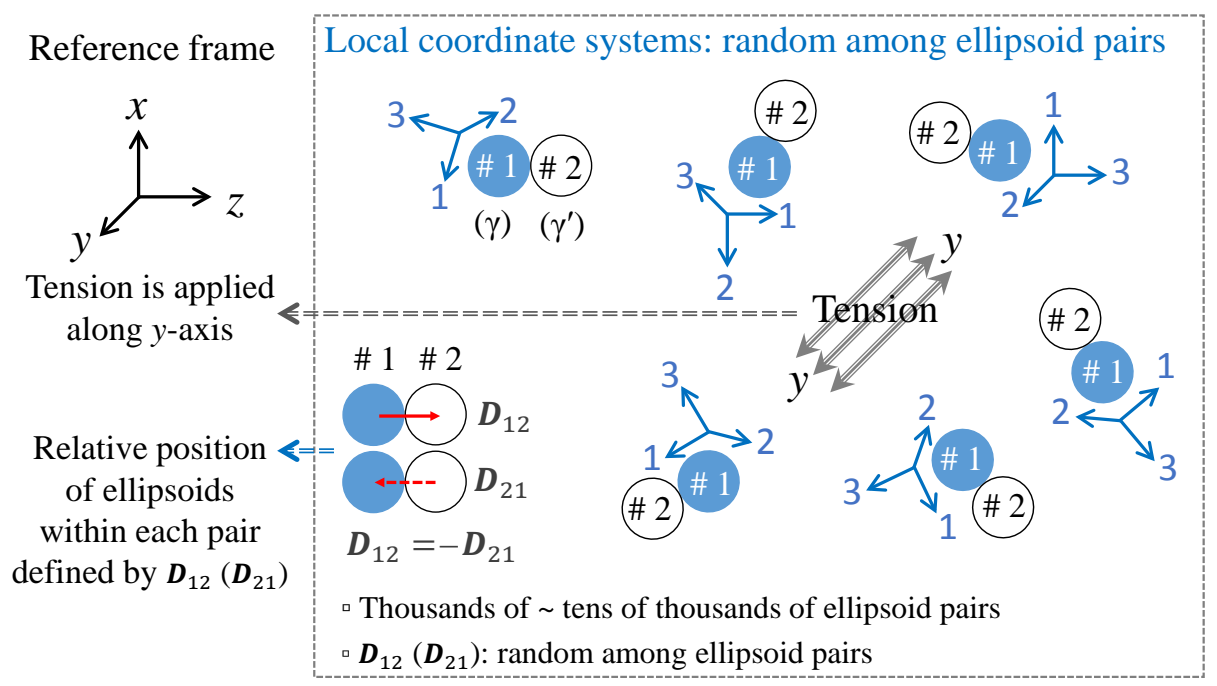

Fig. 1. Reference and local coordinate systems, and schematic 2-D representation of ellipsoid pairs with applied tension along $y$-axis. In application to superalloys that follows, phase \# 1 is $\gamma$ and \# 2 is $\gamma^{\prime}$.

\subsubsection{An approximate finite strain algorithm}

Similar to Neil et al. [24], the Jaumann derivative of the Cauchy stress rate $\hat{\boldsymbol{\sigma}}_{c}$ is introduced to account for local co-rotation (Eq. (16)) or crystal lattice co-rotation (Eq. (17)). The constitutive rules for both grain-level (Eq.(1/a)) and macroscopic scale (Eq.(8)) are rewritten in terms of the local co-rotation or crystal lattice co-rotation stress rates:

$$
\begin{gathered}
\hat{\boldsymbol{\sigma}}_{\mathrm{c}}=\mathbf{L}_{\mathrm{c}}: \boldsymbol{d}_{\mathrm{c}} \\
\hat{\boldsymbol{\sigma}}=\mathbf{L}: \boldsymbol{d}
\end{gathered}
$$

In this approximate approach, the SC Eqs.(1)-(9) are modified with replacing $\dot{\boldsymbol{\sigma}}, \dot{\boldsymbol{\sigma}}_{c}, \dot{\boldsymbol{\epsilon}}$, and $\dot{\boldsymbol{\epsilon}}_{c}$ by $\hat{\boldsymbol{\sigma}}, \hat{\boldsymbol{\sigma}}_{c}, \boldsymbol{d}$, and $\boldsymbol{d}_{c}$, respectively. After solving the ellipsoidal inclusion equilibrium equation and reaching convergence, all Cauchy stress rates are updated to the reference coordinate system using the definition of the Jaumann derivative as follows:

$$
\begin{gathered}
\dot{\boldsymbol{\sigma}}_{c}=\hat{\boldsymbol{\sigma}}_{c}+\dot{\boldsymbol{\omega}}_{c}^{\text {lat }} \boldsymbol{\sigma}_{c}-\boldsymbol{\sigma}_{c} \dot{\boldsymbol{\omega}}_{c}^{\text {lat }} \\
\dot{\boldsymbol{\sigma}}=\hat{\boldsymbol{\sigma}}+\dot{\boldsymbol{\Omega}} \boldsymbol{\sigma}-\boldsymbol{\sigma} \dot{\boldsymbol{\Omega}}
\end{gathered}
$$

where $\dot{\boldsymbol{\omega}}_{c}^{\text {lat }}$ should be replaced by $\dot{\boldsymbol{\omega}}_{\text {corot }}^{\text {lat }}(\mathrm{Eq} \cdot 17)$ for co-rotation scheme.

\subsection{The update of the material state}

After reaching self-consistency, the finite strain approach introduces additional updates:

(1) Deformation and reorientation of ellipsoids: In the 1-site model [23, 24], a single ellipsoid is used to describe the shape of all inclusions, and macroscopic velocity gradient $\boldsymbol{l}$ is used to update the shape of the average ellipsoid. In the 2-site model, we allow each ellipsoid to deform individually with the constraint from its partner in the pair. The deformation gradient of each ellipsoid within a pair is defined as:

$$
\boldsymbol{F}_{c}^{i+1}=\left(\boldsymbol{I}+\boldsymbol{l}_{\mathrm{ave}}^{i} \Delta t\right) \cdot \boldsymbol{F}_{c}^{i}
$$


where $\boldsymbol{l}_{\text {ave }}^{i}$ is the weighted average velocity gradient of the two ellipsoids within a pair at the $i$-th deformation step, taking the form

$$
\boldsymbol{l}_{\mathrm{ave}}=w_{1} \boldsymbol{l}_{1}+w_{2} \boldsymbol{l}_{2}
$$

where $w_{1}$ and $w_{2}$ are the weights of each ellipsoid determined by the volume fractions of each phase. It should be noted that $\boldsymbol{l}_{\text {ave }}=\boldsymbol{d}_{\text {ave }}+\dot{\boldsymbol{\omega}}_{\text {ave }}$ with $\boldsymbol{d}_{\text {ave }}=w_{1} \boldsymbol{d}_{1}+w_{2} \boldsymbol{d}_{2}$, and the contribution of $\dot{\boldsymbol{\omega}}_{\text {ave }}$ is taken into account in a separate step (see Item (2) below). The same as the 1-site approach [23, 24], the updated shape and orientation of the ellipsoid is given by the square root of eigenvalues and eigenvectors of $\boldsymbol{F} \cdot \boldsymbol{F}^{\mathrm{T}}$.

(2) Rotations: Rodrigues' rotation formula is used for each pair to update the morphological orientation with $\dot{\boldsymbol{\omega}}_{\text {ave }}$ (Eq. 15$)$ ) and the crystallographic orientation with $\dot{\boldsymbol{\omega}}_{c}^{\text {lat }}$ (Eq. 16. $)$ for local-co-rotation scheme and $\dot{\boldsymbol{\omega}}_{\text {corot }}^{\text {lat }}(\mathrm{Eq} \cdot(17))$ for co-rotation scheme.

(3) The relative position of the two ellipsoids within a pair: $\boldsymbol{D}_{12}^{i}$ is also rotated with $\dot{\boldsymbol{\omega}}_{\text {ave }}$ (Eq. (15)), i.e. it follows the rotation of the local coordinate system. Furthermore, the distance between the two ellipsoids $\left|\boldsymbol{D}_{12}^{i}\right|$ is computed as the summation of the average length of the three principal axes of ellipsoid \# 1 and that of ellipsoid \# 2 .

\section{Results}

\subsection{Application to superalloys}

Ni-based superalloys are composed of $\gamma$ matrix and $\gamma^{\prime}$ precipitates, which have coherent or semi-coherent interface and display an exact cube-cube orientation relationship [32], making them suitable for engineering applications at elevated temperatures. Recently, a new generation of Ni-based superalloys has been developed containing a higher volume fraction of about $50 \%$ of $\gamma^{\prime}$ precipitates, of which the macroscopic and microscopic mechanical properties and the deformation mechanisms at ambient and elevated temperatures have been systematically characterized [10, 12, 13]. Here we extracted the in situ neutron diffraction experimental data of Alloy $720 \mathrm{Li}$ with a microstructure of two randomly intermixed size distribution of $\gamma^{\prime}$ precipitates at $20{ }^{\circ} \mathrm{C}, 400{ }^{\circ} \mathrm{C}, 500{ }^{\circ} \mathrm{C}$ and $650{ }^{\circ} \mathrm{C}$ from Daymond et al. [10], and Alloy RR1000 with fine, medium, and coarse $\gamma^{\prime}$ microstructures at $20{ }^{\circ} \mathrm{C}$ from Grant et al. 12 and $750{ }^{\circ} \mathrm{C}$ from Francis et al. [13] to verify our model. All the experiments were performed under uniaxial tension.

The numerical code employed in this work is a 2-site update of the 1-site EPSC code (Version 4) developed by Tomé and co-workers written in Fortran 77 [23, 24, 26, 33]. In this model 5000 grain pairs with random initial morphological and crystallographic orientation distributions are chosen. Spherical grains are assumed for both phases at the initial state in the simulation (see microscopy pictures in [10, 12, 13]). As mentioned in Section 2.1.1. one of the limitations of the 2-site algorithm for modeling deformation behaviors of Ni-based superalloys is that there are many coherent $\gamma^{\prime}$ grains embedded in one $\gamma$ grain but only two ellipsoids representing the grain(s) in each phase in the model. Furthermore, although the volume fraction of $\gamma^{\prime}$ phase is the same (45\%), $\gamma^{\prime}$ grain size is not fixed for the fine, medium, and coarse $\gamma^{\prime}$ microstructures in Alloy RR1000. Moreover, the interaction strength between different phases should not be the same, where the exact microstructures of the two phases should be considered. In order to take into account the difference in microstructure (or morphology) of $\gamma^{\prime}$ phase and that between phases, an interaction factor $f_{\gamma-\gamma^{\prime}}=R_{\gamma^{\prime}} / R_{\gamma}$ is introduced to define the interaction strength between $\gamma$ and $\gamma^{\prime}$ grains, where $R_{\gamma^{\prime}}$ and $R_{\gamma}$ are the initial radii of ellipsoids (spheriods) representing $\gamma^{\prime}$ and $\gamma$ grains, respectively. 
The initial morphology of $\gamma$ grains is set to 1:1:1 (ellipsoid's radii), and therefore that of $\gamma^{\prime}$ grains is $f_{\gamma-\gamma^{\prime}}: f_{\gamma-\gamma^{\prime}}: f_{\gamma-\gamma^{\prime}}$. Ellipsoids representing both phases are set equal-sized, i.e. $f_{\gamma-\gamma^{\prime}}=1.0$, for the calculations in this section. The effect of changing $f_{\gamma-\gamma^{\prime}}$ will be examined later. The cube-cube orientation relationship of $\gamma-\gamma^{\prime}$ is represented by assigning the same crystallographic orientation to both grains within each pair. A $45 \%$ weighting of $\gamma^{\prime}$ (55\% of $\gamma$ ) is utilized for all the cases fitted in this work. Tension is applied along $y$-axis of the reference coordinate system, as shown in Fig. 1. The elastic constants used and slip systems selected for $\gamma$ and $\gamma^{\prime}$ single crystals at different temperatures follow those employed in Daymond et al. [10. Crystal lattice co-rotation scheme (Eq. 177) is applied to capture the texture evolution. The implemented plastic flow law for a given slip system is an extended Voce hardening law [34, 35], which is described by an evolution of the threshold stress with accumulated shear strain $\Gamma$ in each grain based on the following equation:

$$
\tau^{s}=\tau_{0}^{s}+\left(\tau_{1}^{s}+\theta_{1}^{s} \Gamma\right)\left(1-\exp \left(-\frac{\theta_{0}^{s} \Gamma}{\tau_{1}^{s}}\right)\right)
$$

where $\tau_{0}^{s}, \theta_{0}^{s}, \theta_{1}^{s}$, and $\tau_{0}^{s}+\tau_{1}^{s}$ are the initial critical resolved shear stress (CRSS), the initial hardening rate, the asymptotic hardening rate, and the back-extrapolated CRSS, respectively. The hardening coefficients used in Eq.24) are fitted to give an optimum agreement with the experimental data, which are listed in Table 1. It should be noted that calibrations of the hardening coefficients have been done for all the cases studied by employing both 2-site and 1-site models in this work.

Predicted results are shown in Figs. 2 and 3, which generally fit the experimental data very well. In order to make a thorough comparison between the existing small strain 2-site EPSC model [10] and finite strain 1-site EPSC model [24], we implemented non-rotation scheme in our 2-site model where both ellipsoid and crystal lattice do not rotate, and extended the finite strain 1-site single-phase code [23, 24, 26, 33] to a dual-phase application to Ni-based superalloys utilizing crystal lattice co-rotation scheme. Lattice strains calculated without texture evolution (non-rotation scheme) and using 1-site model (co-rotation) are plotted in Figs. 2 and 3 as a comparison. It is indicated that almost the same results are obtained with and without co-rotation by employing the 2-site model, where only slight deviations are observed after some accumulations of the applied strain $(\epsilon)$. The 1-site model (co-rotation) also gives very similar predictions comparing to the 2 -site model (co-rotation) except the case of Alloy $720 \mathrm{Li}$ at $500{ }^{\circ} \mathrm{C}$ (Fig. 2(e-f)), where large deviation from the experimental data is observed. It is revealing to regard how the two phases deform and share the applied strain for Alloy 720Li at various temperatures to figure out the origin of the differences between models. It is well known that there is a peak strength temperature for Ni-based superalloys below which they display anomalous behavior where the yield stress at high temperature is larger than that at room temperature even though the strength of the $\gamma$ phase decreases with increasing temperature [36]. Below the peak strength temperature, the predominant slip system in $\gamma^{\prime}$ phase is $\{111\}\langle 110\rangle$, while above it $\{100\}\langle 110\rangle$ is more active [37]. Fig. 4 shows evolution of the average number of active systems per grain (AVACS). The 2-site model (with and without co-rotation) shows that the AVACS of $\gamma$ is larger than that of $\gamma^{\prime}$ at $20{ }^{\circ} \mathrm{C}$, but it reverses at 400 and $500{ }^{\circ} \mathrm{C}$ and then reverses back at $600{ }^{\circ} \mathrm{C}$, which indicates that there is load transfer between 20 and $500{ }^{\circ} \mathrm{C}\left(\gamma^{\prime}\right.$ precipitates is the softer phase) and opposite load transfer at higher temperature ( $\gamma$ matrix is softer), as shown in Fig. 5. While the relative value of AVACS of $\gamma$ and $\gamma^{\prime}$ predicted by the 1-site model for the case at $500{ }^{\circ} \mathrm{C}$ is opposite to that predicted by the 2 -site model when $\epsilon>0.08$, which 
is the strain value where the deviations in lattice strains start (Fig. 2(e-f)). As a result, the phase specific strain of $\gamma$ phase predicted by the 1-site model is much higher than that predicted by the 2-site model (Fig. 5(c)). The relative activity of $\{111\}\langle 110\rangle$ and $\{100\}\langle 110\rangle$ slip systems in $\gamma^{\prime}$ phase changing with temperature is also well captured by the 2 -site model. At 400 and $500{ }^{\circ} \mathrm{C}$ (Fig. $4(\mathrm{~d})$ and (f)), $\{111\}\langle 110\rangle$ becomes more active than $\{100\}\langle 110\rangle$ when $\epsilon>0.01$ or so, but $\{100\}\langle 110\rangle$ becomes predominant again when $\epsilon>0.06$ (for 2-stie, co-rotation) or $\epsilon>0.075$ (for 2 -stie, non-rotation) at $500{ }^{\circ} \mathrm{C}$. At $600{ }^{\circ} \mathrm{C},\{100\}\langle 110\rangle$ is predominant. This indicates that $500{ }^{\circ} \mathrm{C}$ is the temperature very close to the peak strength temperature of Alloy 720Li, around which the relative activity of $\{111\}\langle 110\rangle$ and $\{100\}\langle 110\rangle$ slip systems in $\gamma^{\prime}$ phase alternates during plastic deformation. As a comparison, the 1-site model failed to capture such details for the case at $500{ }^{\circ} \mathrm{C}$, where the grain-grain interaction is neglected. Deformed textures predicted by utilizing the 2-site (co-rotation) and 1-site (co-rotation) models for Alloy 720Li are also compared, as shown in Fig. 6, where the pole figures were calculated and plotted using the MTEX open-source package [38, 39]. The two models display similar texture for each case that the $\langle 111\rangle$ direction turning toward $\mathrm{AD}$ (i.e. the tensile stress axis) and $\langle 110\rangle$ direction turning away from it, but the texture strength and the grain orientation distribution are not exactly the same. It is because of the accumulated effect of their difference in deformation mechanism, which starts from $\epsilon=0.03 \sim 0.06$ (Fig. 4(a), (c), (e), and (g)).

Figs. 2-6 demonstrate that the 2-site model (finite strain, co-rotation), 2-site model (small strain, non-rotation), and 1-site model (finite strain, co-rotation) distribute different predictions at relatively larger deformation stage. It is also noticed that the predicted transverse lattice strains are not as good as the axial for the fine, medium, and coarse $\gamma^{\prime}$ microstructures at both $20{ }^{\circ} \mathrm{C}$ and $750{ }^{\circ} \mathrm{C}$ (Fig. 3), where the total deformation is less than 0.1 and 0.04 , respectively. This phenomenon is supposed to originate from the 2 -site formulation (Section 2.1), in which the relative size of ellipsoids within a pair, i.e. $f_{\gamma-\gamma^{\prime}}$ defined above, plays an important role in grain-grain interaction and hence influences the predictions. In what follows, we will investigate the differences in the prediction of lattice strains between models at large deformation state $(\epsilon=0.5)$ and the effects of the interaction strength between $\gamma$ and $\gamma^{\prime}$ grains on the homogenized results by employing the 2 -site model. 
Table 1. Hardening parameters describing the evolution of threshold stress with deformation (Eq. 24 $)$ used for best fitting experimental (Exp.) data in [10, 12, 13, where $\tau_{0}^{s}, \theta_{0}^{s}, \theta_{1}^{s}$, and $\tau_{0}^{s}+\tau_{1}^{s}$ are the initial critical resolved shear stress (CRSS), the initial hardening rate, the asymptotic hardening rate, and the back-extrapolated CRSS, respectively. Parameters in front of " " " are for 2-site model and that behind it are for 1-site model ( i.e. 2-site / 1-site).

\begin{tabular}{|c|c|c|c|c|c|c|c|}
\hline Exp. data & $T$ & Phase & Slip system & $\tau_{0}$ & $\tau_{1}$ & $\theta_{0}$ & $\theta_{1}$ \\
\hline \multirow{11}{*}{$720 \mathrm{Li}$ [10] } & \multirow{2}{*}{$20{ }^{\circ} \mathrm{C}$} & $\gamma$ & $\{111\}\langle 110\rangle$ & $375 / 376$ & $5 / 5$ & $50 / 60$ & $50 / 35$ \\
\hline & & Intermixed $\gamma^{\prime}$ & $\{111\}\langle 110\rangle$ & $385 / 384$ & $5 / 5$ & $780 / 798$ & $780 / 754$ \\
\hline & \multirow{3}{*}{$400{ }^{\circ} \mathrm{C}$} & \multirow{3}{*}{$\begin{array}{c}\gamma \\
\text { Intermixed } \gamma^{\prime}\end{array}$} & $\{111\}\langle 110\rangle$ & $498 / 498$ & $5 / 5$ & $1207 / 1190$ & $296 / 289$ \\
\hline & & & $\{111\}\langle 110\rangle$ & $437 / 434$ & $5 / 5$ & $294 / 290$ & $145 / 128$ \\
\hline & & & $\{100\}\langle 110\rangle$ & $450 / 444$ & $5 / 5$ & $116 / 121$ & 99 / 85 \\
\hline & \multirow{3}{*}{$500{ }^{\circ} \mathrm{C}$} & \multirow{3}{*}{$\begin{array}{c}\gamma \\
\text { Intermixed } \gamma^{\prime}\end{array}$} & $\{111\}\langle 110\rangle$ & $477 / 473$ & $5 / 5$ & $1040 / 1038$ & $224 / 129$ \\
\hline & & & $\{111\}\langle 110\rangle$ & $444 / 435$ & $5 / 5$ & $283 / 283$ & $128 / 54$ \\
\hline & & & $\{100\}\langle 110\rangle$ & $525 / 527$ & $5 / 5$ & 67 / 72 & $10 / 9$ \\
\hline & \multirow{3}{*}{$650{ }^{\circ} \mathrm{C}$} & \multirow{3}{*}{$\begin{array}{c}\gamma \\
\text { Intermixed } \gamma^{\prime}\end{array}$} & $\{111\}\langle 110\rangle$ & $227 / 227$ & $20 / 20$ & $593 / 596$ & $118 / 110$ \\
\hline & & & $\{111\}\langle 110\rangle$ & $331 / 331$ & $58 / 60$ & $2624 / 2623$ & 399 / 394 \\
\hline & & & $\{100\}\langle 110\rangle$ & $272 / 273$ & $17 / 17$ & 927 / 927 & $157 / 149$ \\
\hline \multirow{6}{*}{ RR1000 [12] } & \multirow{6}{*}{$20{ }^{\circ} \mathrm{C}$} & $\gamma$ & $\{111\}\langle 110\rangle$ & $410 / 411$ & $11 / 10$ & 1203 / 1192 & $122 / 110$ \\
\hline & & Fine $\gamma^{\prime}$ & $\{111\}\langle 110\rangle$ & $426 / 412$ & $41 / 50$ & 1919 / 1850 & $372 / 350$ \\
\hline & & $\gamma$ & $\{111\}\langle 110\rangle$ & $378 / 379$ & $6 / 6$ & $1832 / 2379$ & $73 / 55$ \\
\hline & & Medium $\gamma^{\prime}$ & $\{111\}\langle 110\rangle$ & $391 / 391$ & $8 / 10$ & 1886 / 1867 & $1235 / 1228$ \\
\hline & & $\gamma$ & $\{111\}\langle 110\rangle$ & $291 / 291$ & $10 / 10$ & $1223 / 1237$ & 238 / 224 \\
\hline & & Coarse $\gamma^{\prime}$ & $\{111\}\langle 110\rangle$ & $376 / 376$ & $10 / 11$ & $1693 / 1650$ & $1384 / 1374$ \\
\hline \multirow{9}{*}{ RR1000 [13. } & \multirow{9}{*}{$750{ }^{\circ} \mathrm{C}$} & $\gamma$ & $\{111\}\langle 110\rangle$ & $268 / 268$ & $10 / 10$ & $55 / 56$ & $41 / 37$ \\
\hline & & & $\{111\}\langle 110\rangle$ & $319 / 315$ & $10 / 10$ & 2058 / 2062 & $95 / 88$ \\
\hline & & मान् $\gamma$ & $\{100\}\langle 110\rangle$ & $278 / 279$ & $10 / 10$ & 100 / 98 & $50 / 45$ \\
\hline & & $\gamma$ & $\{111\}\langle 110\rangle$ & $195 / 195$ & $10 / 10$ & $988 / 1004$ & 87 / 76 \\
\hline & & Mediuun & $\{111\}\langle 110\rangle$ & $247 / 247$ & $20 / 20$ & 2843 / 2879 & 1718 / 1691 \\
\hline & & viedintis & $\{100\}\langle 110\rangle$ & $217 / 218$ & $10 / 10$ & $1280 / 1304$ & $285 / 274$ \\
\hline & & $\gamma$ & $\{111\}\langle 110\rangle$ & $155 / 153$ & $21 / 21$ & 1029 / 1036 & $93 / 53$ \\
\hline & & Coarc & $\{111\}\langle 110\rangle$ & $148 / 148$ & $177 / 189$ & 36,739 / 36,140 & $995 / 973$ \\
\hline & & coarse & $\{100\}\langle 110\rangle$ & $166 / 164$ & 84 / 82 & 8287 / 8016 & $664 / 631$ \\
\hline
\end{tabular}



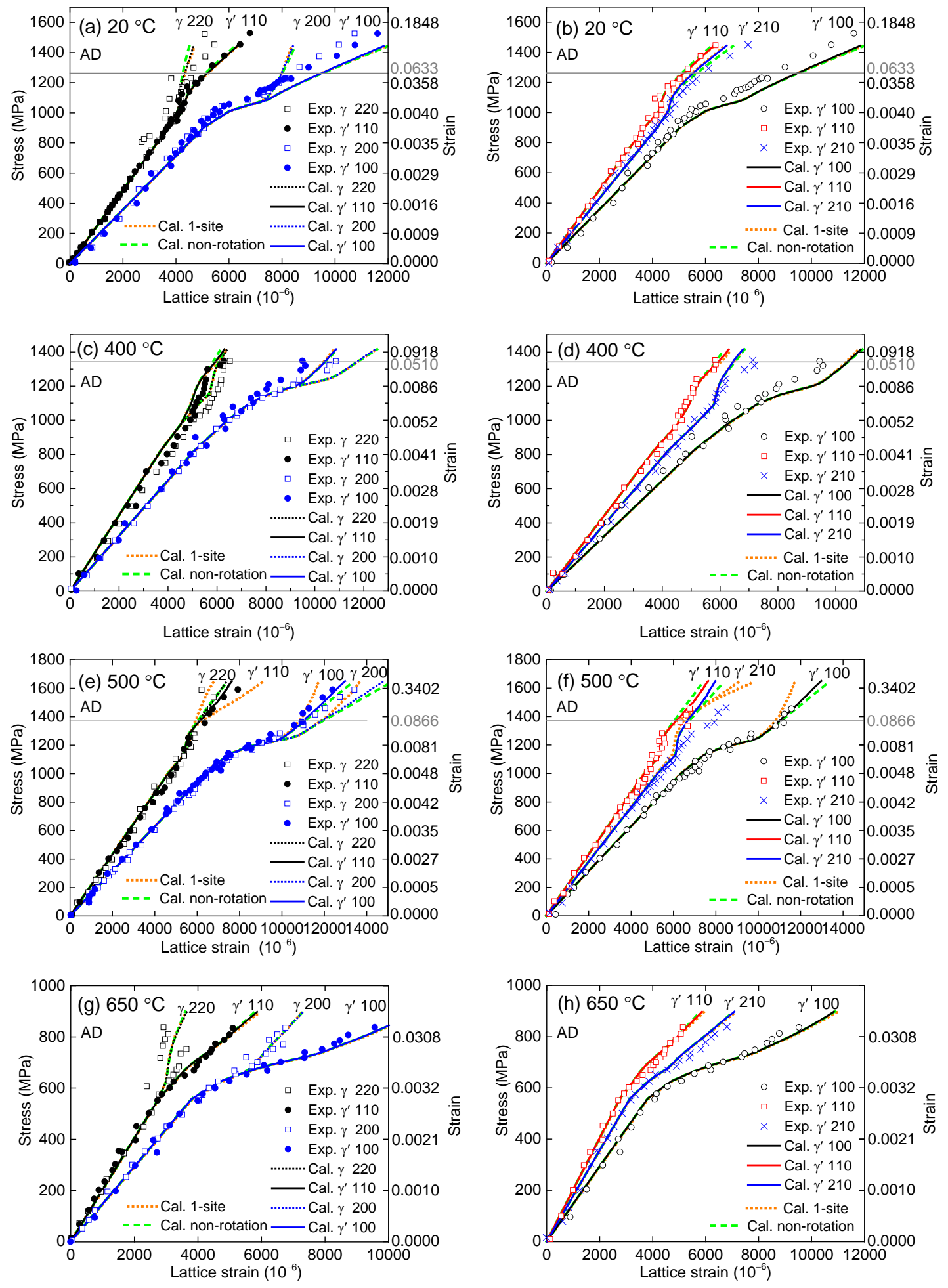

Fig. 2. Comparison of axial lattice strains measured (points, data from Daymond et al. [10]) and simulated (lines) for Alloy $720 \mathrm{Li}$ with intermixed $\gamma^{\prime}$ precipitates at (a-b) $20{ }^{\circ} \mathrm{C}$, (c-d) $400{ }^{\circ} \mathrm{C}$, (e-f) $500{ }^{\circ} \mathrm{C}$, and (g-h) 650 ${ }^{\circ} \mathrm{C}$. Results of non-rotation scheme (2-site model) and 1-site model (co-rotation) are shown as a comparison. Strains corresponding to the applied stresses on the left axis are labelled on the right axis for each panel. 

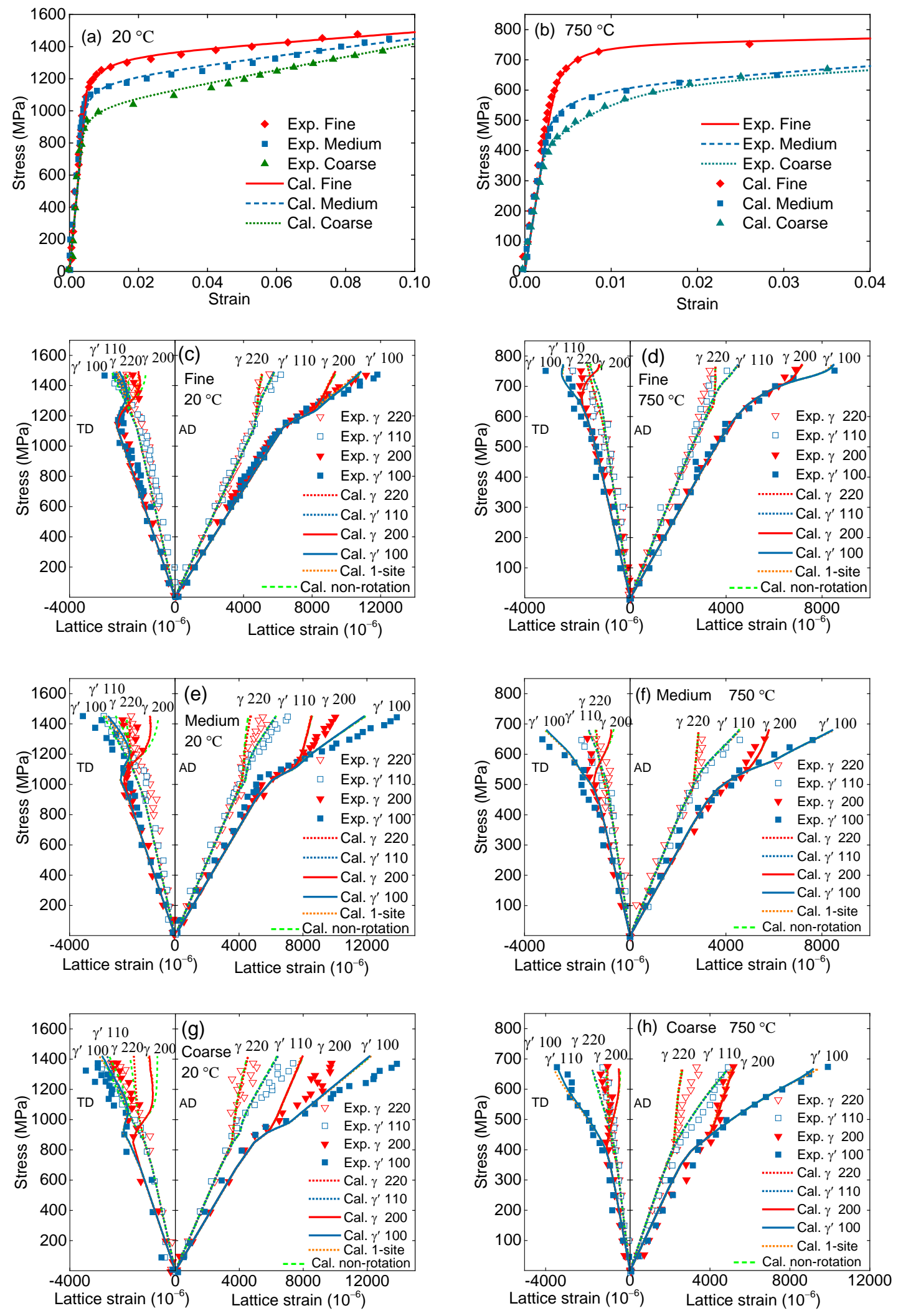

Fig. 3. Comparison of (a-b) stress-strain curves and (c-h) lattice strains measured (points, data from Grant et al. [12] and Francis et al. [13]) and simulated (lines) for Alloy RR1000 with different $\gamma^{\prime}$ microstructures at $20{ }^{\circ} \mathrm{C}$ and $750{ }^{\circ} \mathrm{C}$. Results of non-rotation scheme (2-site model) and 1-site model (co-rotation) are shown as a comparison. 

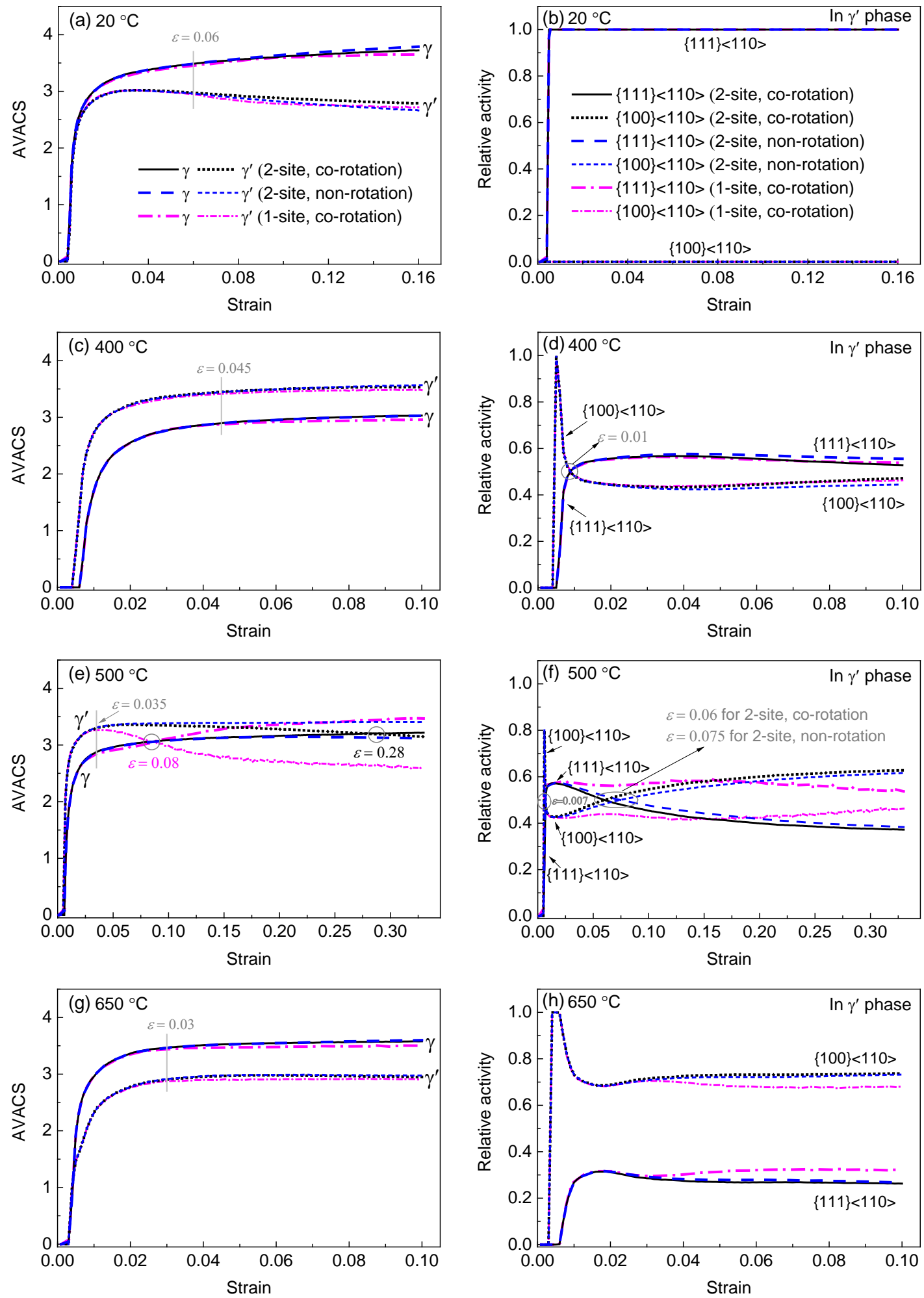

Fig. 4. Evolution of AVACS and relative activity of $\{111\}\langle 110\rangle$ and $\{100\}\langle 110\rangle$ slip systems in $\gamma^{\prime}$ phase for Alloy $720 \mathrm{Li}$ at $(\mathrm{a}-\mathrm{b}) 20{ }^{\circ} \mathrm{C},(\mathrm{c}-\mathrm{d}) 400{ }^{\circ} \mathrm{C}$, (e-f) $500{ }^{\circ} \mathrm{C}$, and $(\mathrm{g}-\mathrm{h}) 650{ }^{\circ} \mathrm{C}$ predicted by the 2 -site model (co-rotation), 2-site model (non-rotation), and 1-site model (co-rotation). 


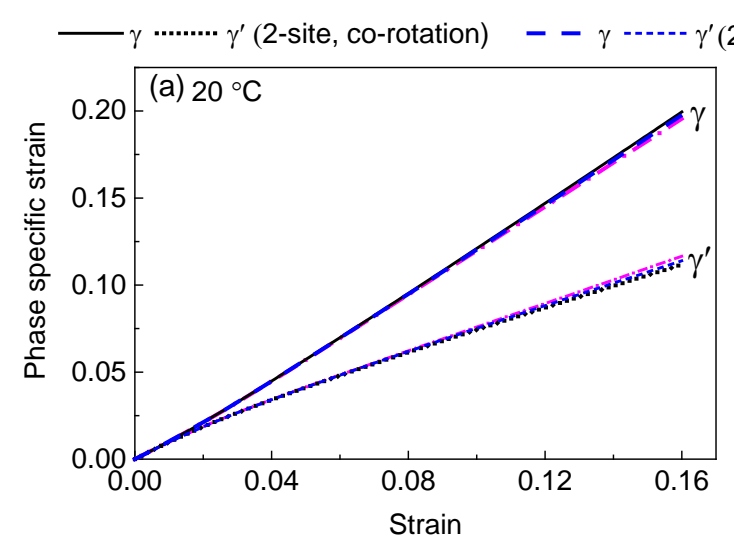

(2-site, non-rotation) $\quad-\cdot-\gamma-\gamma^{-\cdot-\cdot-\cdot} \gamma^{\prime}$ (1-site, co-rotation)
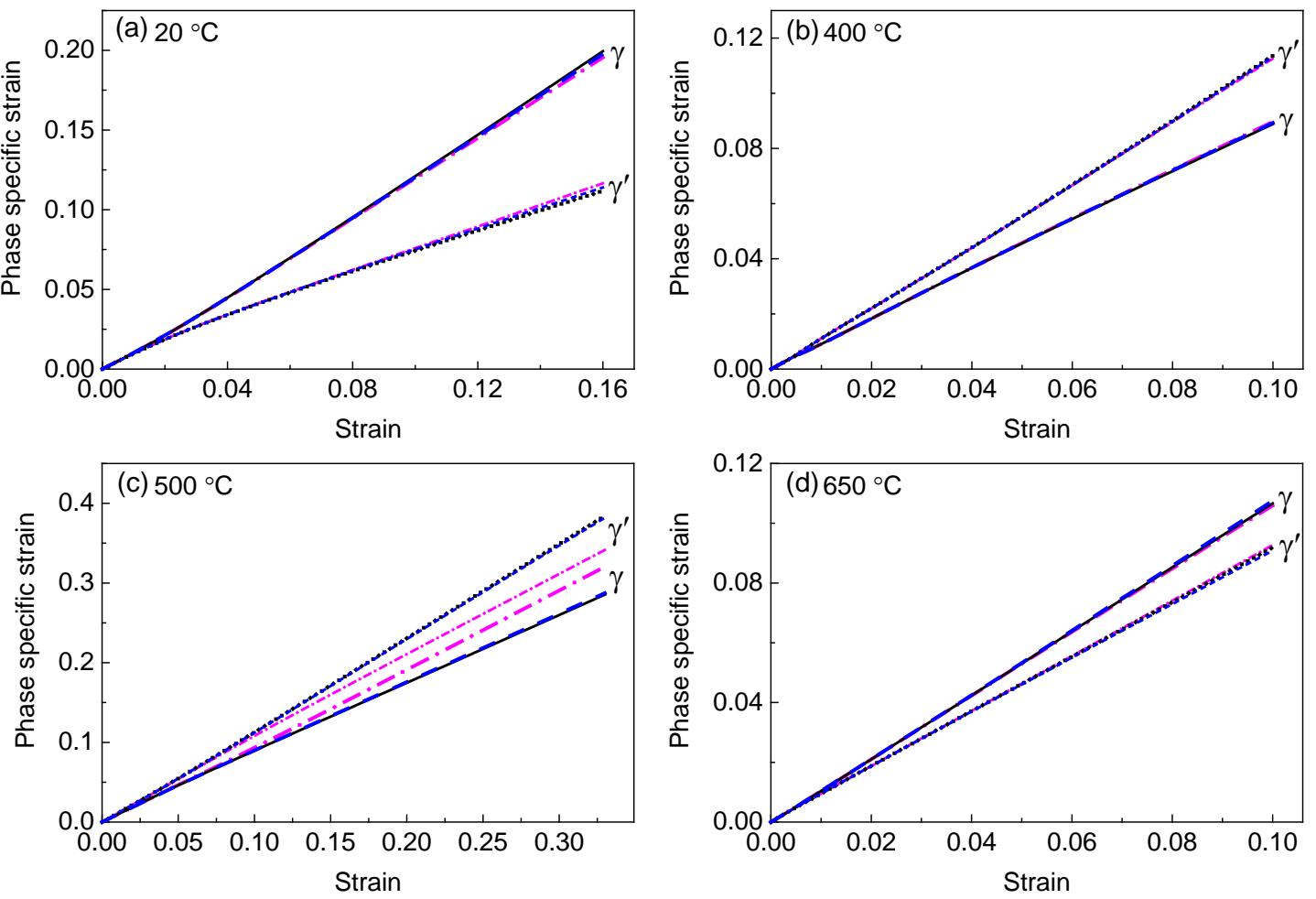

Fig. 5. Phase specific strains for Alloy $720 \mathrm{Li}$ at (a) $20{ }^{\circ} \mathrm{C}$, (b) $400{ }^{\circ} \mathrm{C}$, (c) $500{ }^{\circ} \mathrm{C}$, and (d) $650{ }^{\circ} \mathrm{C}$ predicted by the 2 -site model (co-rotation), 2 -site model (non-rotation), and 1-site model (co-rotation). 
(a) The initial $\quad \gamma / \gamma^{\prime}$
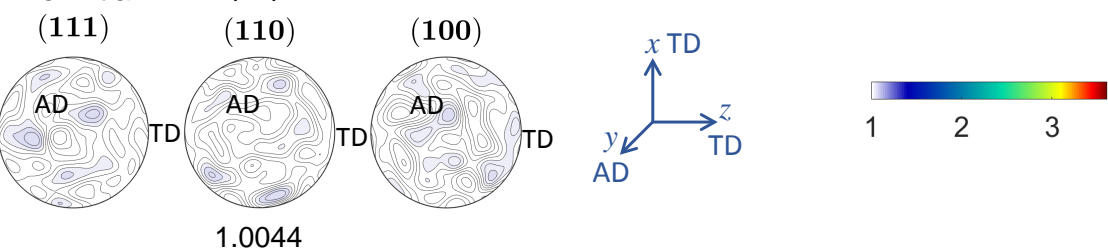

2-site, co-rotation $\left(\gamma / \gamma^{\prime}\right)$

1-site, co-rotation $\left(\gamma / \gamma^{\prime}\right)$

(b) $\varepsilon=0.16,20^{\circ} \mathrm{C}$

(111)

(110)

(100)

(c) $\varepsilon=0.16,20^{\circ} \mathrm{C}$

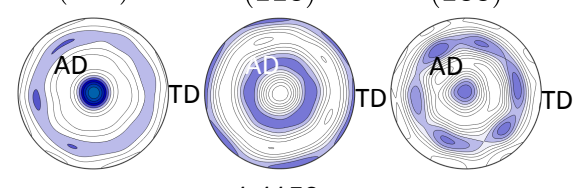

1.1159

(d) $\varepsilon=0.10,400{ }^{\circ} \mathrm{C}$
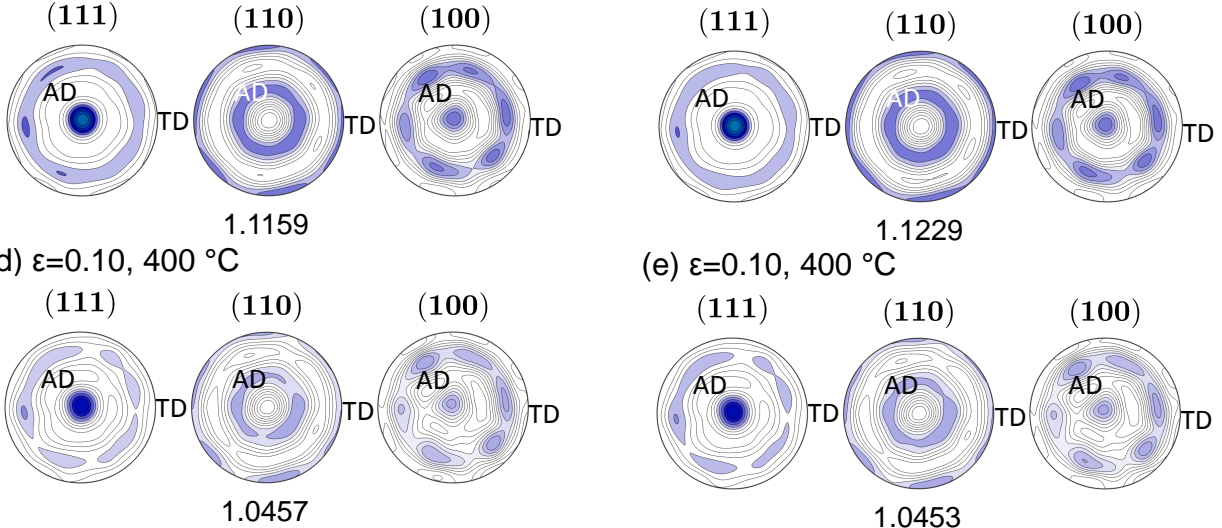

(e) $\varepsilon=0.10,400{ }^{\circ} \mathrm{C}$

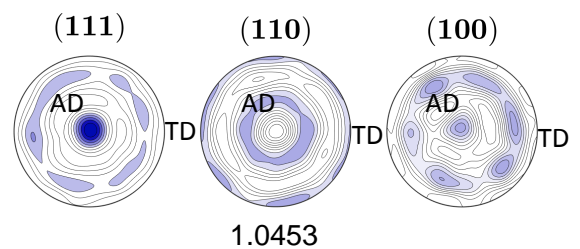

(f) $\varepsilon=0.33,500{ }^{\circ} \mathrm{C}$

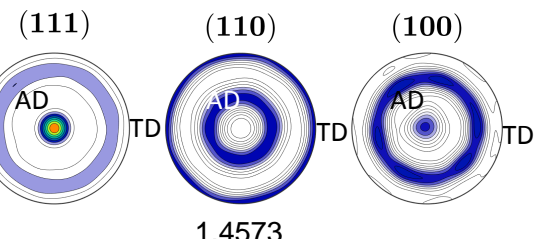

(h) $\varepsilon=0.10,650{ }^{\circ} \mathrm{C}$

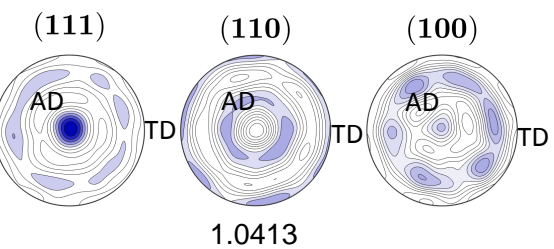

(g) $\varepsilon=0.33,500^{\circ} \mathrm{C}$

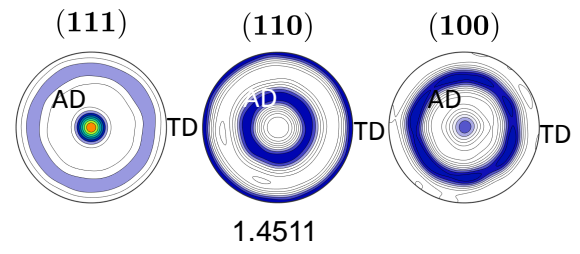

(i) $\varepsilon=0.10,650^{\circ} \mathrm{C}$

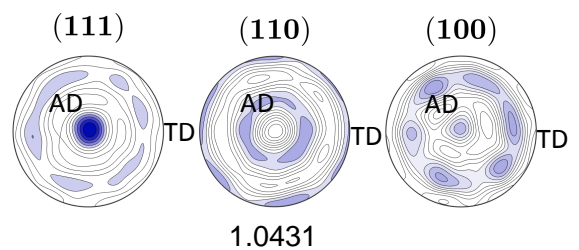

Fig. 6. Pole figures of final deformation state predicted by employing the 2-site (co-rotation) and 1-site (co-rotation) models for Alloy $720 \mathrm{Li}$ at (b-c) $20{ }^{\circ} \mathrm{C}$, (d-e) $400{ }^{\circ} \mathrm{C}$, (f-g) $500{ }^{\circ} \mathrm{C}$, and (h-i) $650{ }^{\circ} \mathrm{C}$. The initial texture is shown in panel (a) as a comparison. Texture index is labelled under each pole figure. AD and TD stand for axial direction and transverse direction, respectively.

\subsection{Comparison between models}

We selected six cases from Section 3.1, i.e. Alloy RR1000 with fine, medium, and coarse $\gamma^{\prime}$ microstructures at $20^{\circ} \mathrm{C}$ and $750{ }^{\circ} \mathrm{C}$, and stretched them to a larger strain of 0.5 for a general comparison to study the differences in the prediction of (both axial and transverse) lattice strains by employing the three models. It should be noticed that there is one more slip type in $\gamma^{\prime}$ phase at $750{ }^{\circ} \mathrm{C}$ than that at $20{ }^{\circ} \mathrm{C}$ (see Table 1 for more details). Deviations in lattice strains along $\mathrm{AD}$ start from $\epsilon=0.10 \sim 0.18$ while that along TD occur from a smaller strain of $\epsilon=0.04 \sim 0.05$, as shown in Fig. 7. The predicted transverse lattice strains by utilizing non-rotation scheme (2-site) are quite different from that with co-rotation scheme (both 2site and 1-site), especially when $\epsilon$ is larger than $0.30 \sim 0.37$ at both 20 and $750{ }^{\circ} \mathrm{C}$. A further 
comparison between 1-site model (co-rotation) and 2-site model (co-rotation) indicates that deviations are anisotropic in crystalline planes and different at the two temperatures. At $20{ }^{\circ} \mathrm{C}$, the differences in lattice strains of $\gamma(200)$ and/or $\gamma^{\prime}(100)$ are much larger than that of $\gamma(220)$ and $\gamma^{\prime}$ (110) (Fig. 7(a), (c), and (e)), while deviation in lattice strain of $\gamma^{\prime}(110)$ also becomes larger at $750{ }^{\circ} \mathrm{C}$ (Fig. 7(b), (d), and (f)). This can be understood from the perspective of grain-grain interaction and its effect on the deformation mechanisms (of $\gamma^{\prime}$ phase), as discussed in next section (Section 3.3. .
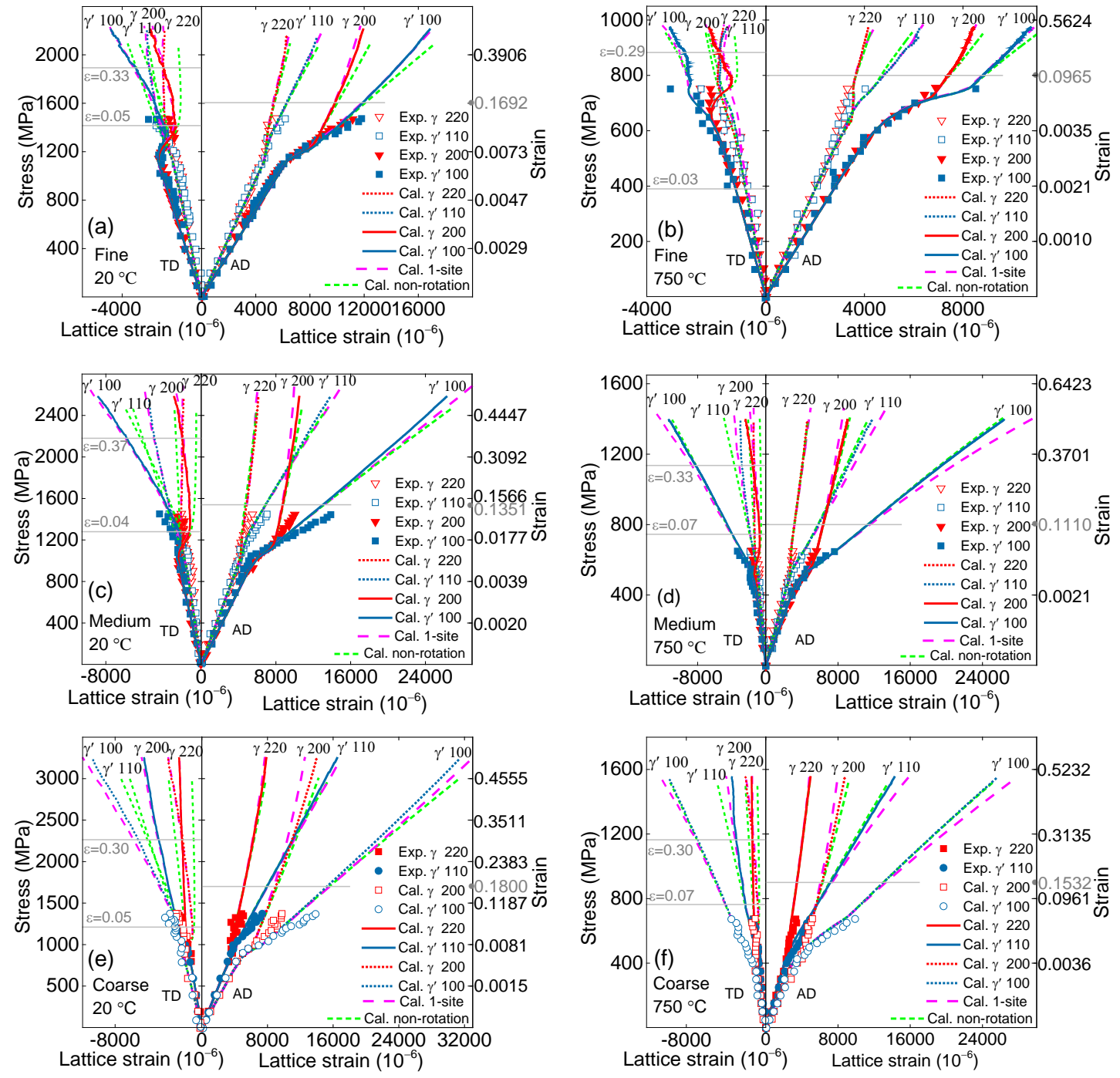

Fig. 7. Comparison between the 2-site model (co-rotation), 2-site model (non-rotation), and 1-site model (co-rotation) on the prediction of lattice strains for Alloy RR1000 with (a-b) fine, (c-d) medium, and (e-f) coarse $\gamma^{\prime}$ microstructures stretched up to 0.5 true strain at $20{ }^{\circ} \mathrm{C}$ and $750{ }^{\circ} \mathrm{C}$. Points are experimental data from Grant et al. [12] and Francis et al. [13].

\subsection{Effects of interaction factor $f_{\gamma-\gamma^{\prime}}$}

The 1-site model (co-rotation) gives different lattice strains from the 2-site model (corotation) when the accumulated strain reaches a critical value, and the differences depend on both crystalline plane and temperature, as discussed in Section 3.2, in which the graingrain interaction is not incorporated. Therefore, it is revealing to know how the grain-grain 
interaction affects the homogenized result in the 2-site model. In our implementation, an interaction factor $f_{\gamma-\gamma^{\prime}}$ is introduced (see Section 3.1) to simulate the interaction strength between the two grains within a pair from the two phases to account for the difference in microstructure (or morphology) of $\gamma^{\prime}$ phase and that between phases. It should be noted that it is a fitting parameter in the model and not relevant to the volume fractions of the two phases. $w_{1}=0.55$ and $w_{2}=0.45$ are used in Eqs.(15) and (23) for all the superalloys studied in this work, which are fixed parameters in the calculation. In this section, cases utilized in Section 3.1 were selected to perform a systematic study on the effects of $f_{\gamma-\gamma^{\prime}}$ on the calculated lattice strains, including Alloy RR1000 with fine, medium, and coarse $\gamma^{\prime}$ microstructures at $20{ }^{\circ} \mathrm{C}$ from Grant et al. [12] and $750{ }^{\circ} \mathrm{C}$ from Francis et al. [13]. We keep all the input parameters the same as those used in Section 3.1 except $f_{\gamma-\gamma^{\prime}}$. Co-rotation scheme is employed (i.e. 2-site model (co-rotation)). $f_{\gamma-\gamma^{\prime}}$ is changed from 1.0 (originally used in Section 3.1) to decreased or increased values to study effects of the interaction strength between $\gamma$ and $\gamma^{\prime}$ grains on the predicted results.

Cases of $f_{\gamma-\gamma^{\prime}}=0.01,0.1,10.0,100.0$ are compared to the case of $f_{\gamma-\gamma^{\prime}}=1.0$. Calculated results are shown in Figs. 8 and 9. Significant differences of lattice strains along both AD and TD are observed by decreasing or increasing $f_{\gamma-\gamma^{\prime}}$ at both temperatures. Deviations in the elastic part of the lattice strains along both $\mathrm{AD}$ and TD are found when $f_{\gamma-\gamma^{\prime}}$ is smaller than 0.1 or larger than 10.0 or so. $f_{\gamma-\gamma^{\prime}}$ has the greatest impact on the transverse lattice strains of $\gamma(200)$ and $\gamma^{\prime}(100)$ at plastic deformation stage for Alloy RR1000 with different $\gamma^{\prime}$ microstructures at $20{ }^{\circ} \mathrm{C}$ (Fig. 8), but only slight effects on that at $750{ }^{\circ} \mathrm{C}$ (Fig. 9). It is apparent that $f_{\gamma-\gamma^{\prime}}$ has great influences on the simulated lattice strains, which also depends on plastic deformation mechanisms for the prediction of the plastic part of lattice strains. In order to dig into it further, evolution of AVACS and relative activity of $\{111\}\langle 110\rangle$ and $\{100\}\langle 110\rangle$ slip systems in $\gamma^{\prime}$ phase are compared for different $f_{\gamma-\gamma^{\prime}}$ values, as shown in Fig. 10. A general conclusion for all the selected cases is that the AVACS in $\gamma^{\prime}$ phase increases with increasing $f_{\gamma-\gamma^{\prime}}$ value. The relative activity of $\{100\}\langle 110\rangle$ in $\gamma^{\prime}$ phase with decreased or increased $f_{\gamma-\gamma^{\prime}}$ values decreases comparing to the case with $f_{\gamma-\gamma^{\prime}}=1.0$ for medium and coarse $\gamma^{\prime}$ microstructures at $750{ }^{\circ} \mathrm{C}$, but different deviations in relative activity are observed for the fine $\gamma^{\prime}$ microstructure where the relative activity of $\{111\}\langle 110\rangle$ and $\{100\}\langle 110\rangle$ alternates during deformation. It hence can be concluded that the effect of $f_{\gamma-\gamma^{\prime}}$ on the deformation mechanisms influences the evolution of grain orientation distribution (Eq. 14p), which influences the predicted lattice strains as a consequence. For high temperature case, there is one more type of cube slip system $(\{100\}\langle 110\rangle)$ activated, which plays a predominant role in the crystal lattice rotation and makes the response to $f_{\gamma-\gamma^{\prime}}$ changing different from that at room temperature.

Since $\gamma-\gamma^{\prime}$ interaction is also affected by the plastic deformation mechanism, i.e. shear across $\gamma / \gamma^{\prime}$ boundaries for fine $\gamma^{\prime}$ microstructure and looping around $\gamma^{\prime}$ grains for medium and coarse microstructures, as discussed in Section 2.1.2, we also studied the effect of $f_{\gamma-\gamma^{\prime}}$ on the predicted lattice strains by utilizing local-co-rotation scheme (Eq.(16)) and compared the results with that predicted by utilizing the co-rotation scheme (Eq.(17)) presented above. Predicted axial lattice strains with changing $f_{\gamma-\gamma^{\prime}}$ values from 0.01 to 100.0 are almost the same by utilizing the two rotation schemes (not shown here), and only slight differences are observed for the prediction of transverse lattice strains, as shown in Fig. 11 where a comparison is displayed among simulated transverse lattice strains with different $f_{\gamma-\gamma^{\prime}}$ values for Alloy RR1000 with different $\gamma^{\prime}$ microstructures at final deformation states of $\epsilon=0.10$ $\left(20{ }^{\circ} \mathrm{C}\right)$ and $\epsilon=0.04\left(750{ }^{\circ} \mathrm{C}\right)$. 

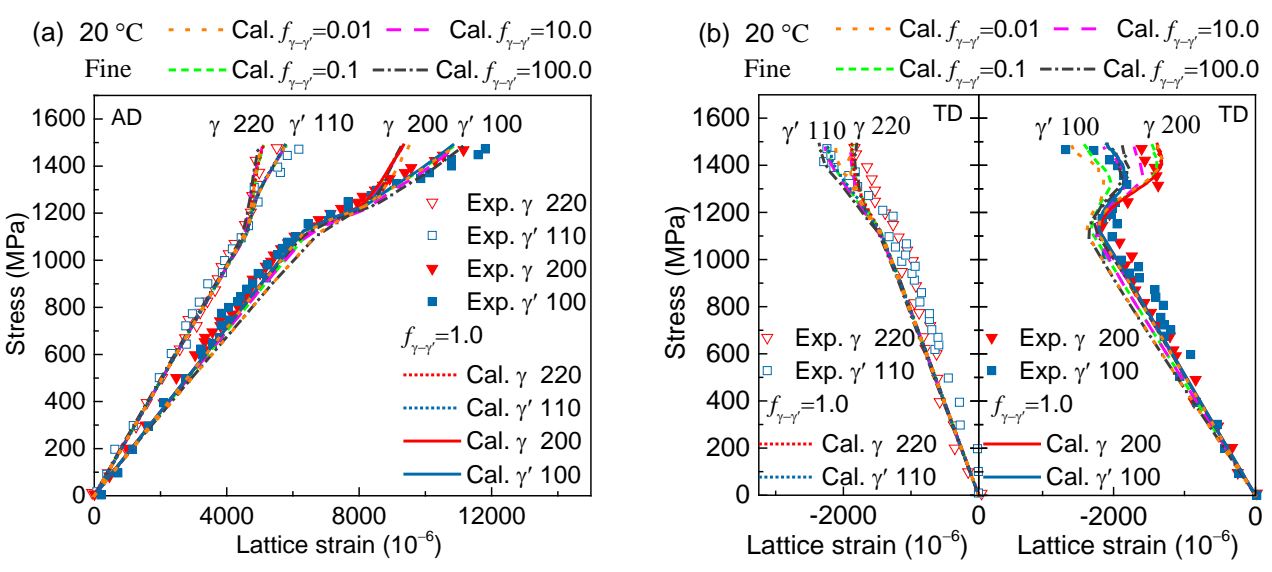

(c) $20{ }^{\circ} \mathrm{C} \quad \cdots$ Cal. $f_{\gamma-\gamma^{\prime}}=0.01--$ Cal. $f_{\gamma-\gamma^{\prime}}=10.0$ Medium - - - - Cal. $f_{y-\gamma^{\prime}}=0.1-\cdot-\cdot$ Cal. $f_{\gamma-\gamma^{\prime}}=100.0$
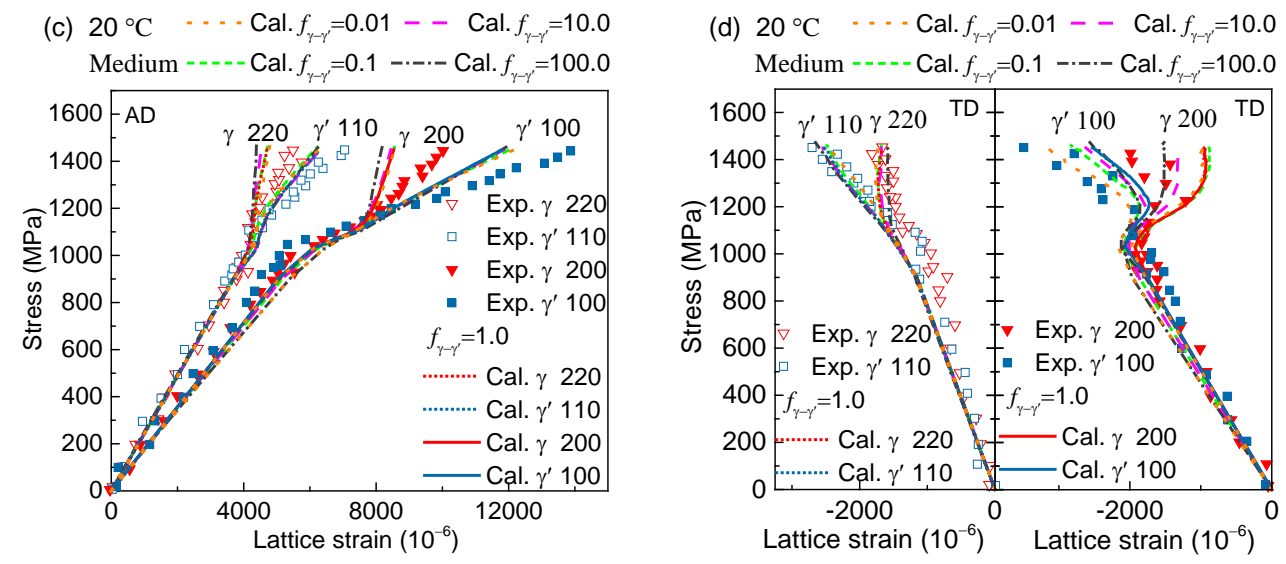

(e) $20^{\circ} \mathrm{C}--$ - Cal. $f_{\gamma-\gamma^{\prime}}=0.01--$ Cal. $f_{\gamma-\gamma^{\prime}}=10.0$
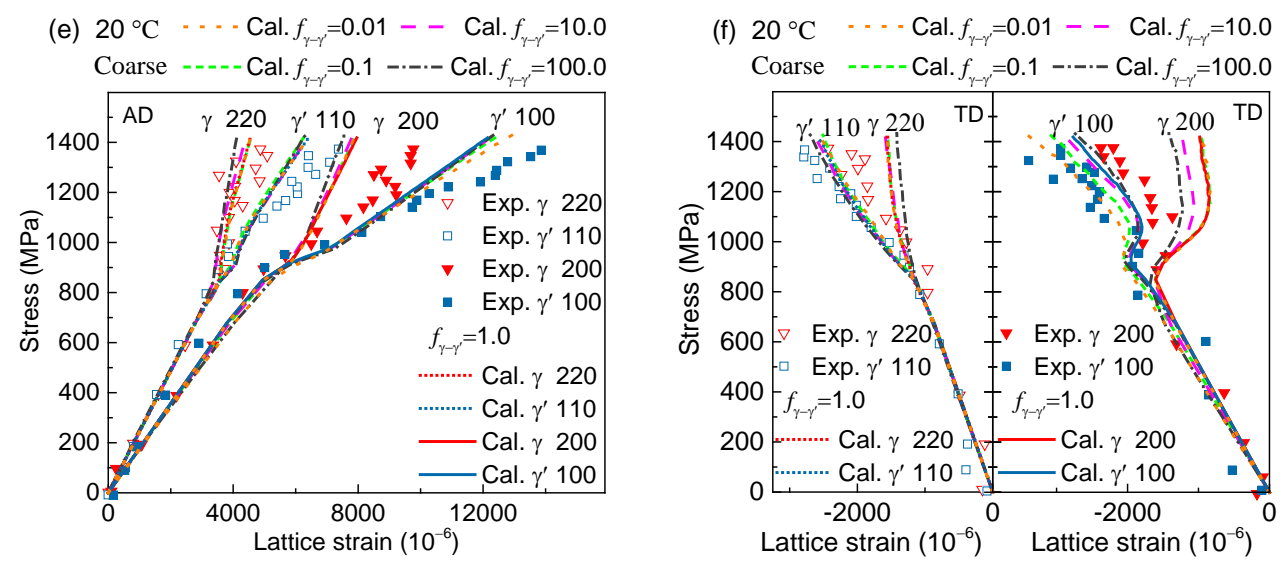

Fig. 8. Comparison among simulated lattice strains with different $f_{\gamma-\gamma^{\prime}}$ values for Alloy RR1000 with different $\gamma^{\prime}$ microstructures at $20{ }^{\circ} \mathrm{C}$ by employing 2-site model (co-rotation). Points are experimental data from Grant et al. [12]. 
(a) $750{ }^{\circ} \mathrm{C}-\cdots$ Cal. $f_{\gamma-\gamma^{\prime}}=0.01-$ Cal. $f_{\gamma-\gamma^{\prime}}=10.0$

Fine $\quad----$ Cal. $f_{y-\gamma}=0.1-\cdot-\cdot$ Cal. $f_{y-\gamma}=100.0$

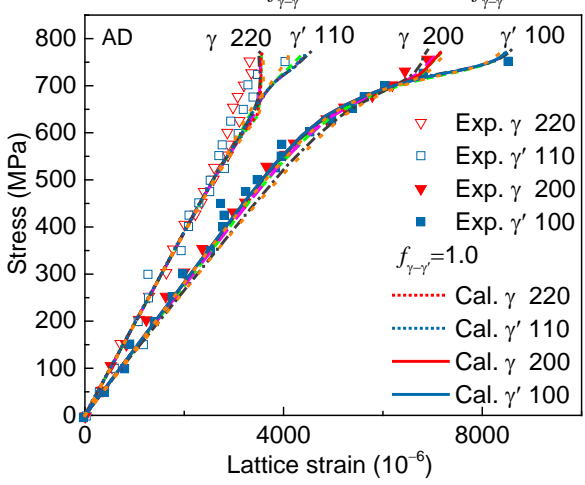

(c) $750{ }^{\circ} \mathrm{C} \cdots-$ Cal. $f_{\gamma-\gamma^{\prime}}=0.01--$ Cal. $f_{\gamma-\gamma^{\prime}}=10.0$

Medium - - - Cal. $f_{\gamma-\gamma=}=0.1$-.-.- Cal. $f_{\gamma-\gamma=}=100.0$

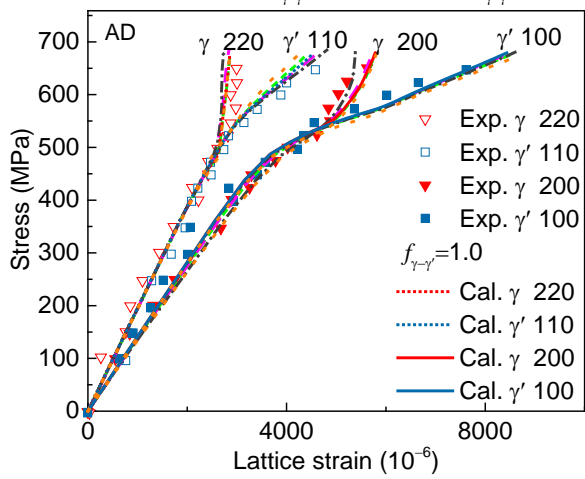

(e) $750{ }^{\circ} \mathrm{C} \mathrm{\cdots} \mathrm{-} \mathrm{Cal.} f_{\gamma-\gamma^{\prime}}=0.01--$ Cal. $f_{\gamma-\gamma^{\prime}}=10.0$ Coarse - - - Cal. $f_{\gamma-\gamma^{\prime}}=0.1-\cdot-\cdot-$ Cal. $f_{\gamma-\gamma^{\prime}}=100.0$

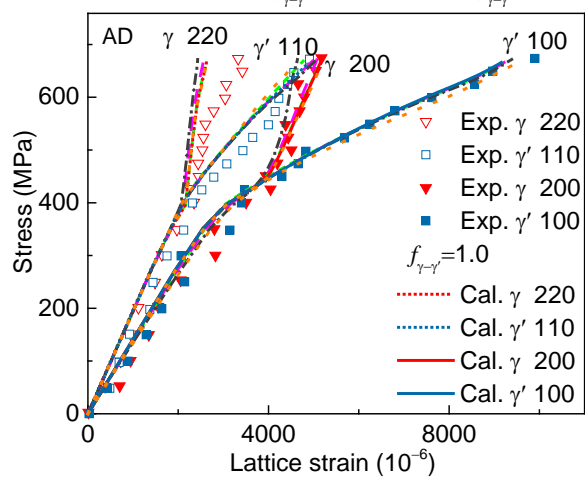

(b) $750{ }^{\circ} \mathrm{C} \cdots$ - Cal. $f_{\gamma-\gamma}=0.01--$ Cal. $f_{\gamma-\gamma}=10.0$

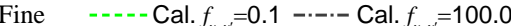

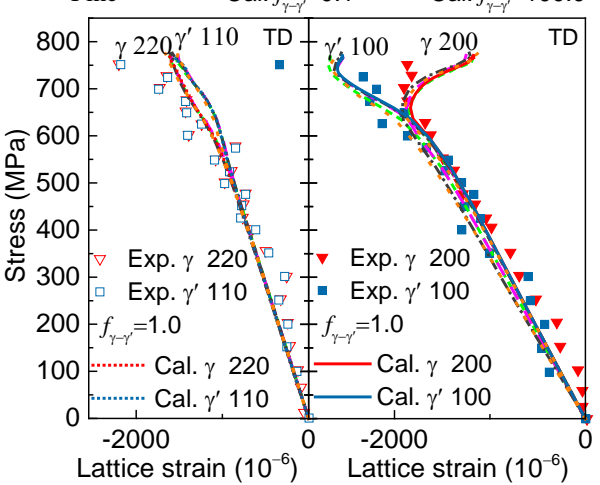

(d) $750{ }^{\circ} \mathrm{C}-\cdots$ - Cal. $f_{\gamma-\gamma^{\prime}}=0.01--$ Cal. $f_{\gamma-\gamma^{\prime}}=10.0$

Medium - - - - Cal. $f_{\gamma-\gamma}=0.1$--.-- Cal. $f_{\gamma-\gamma}=100.0$

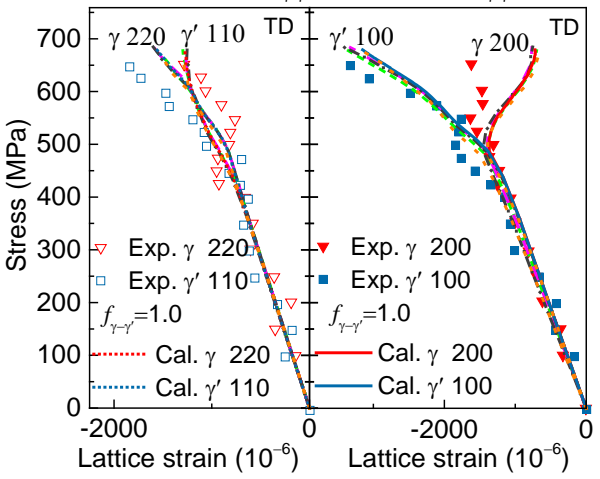

(f) $750{ }^{\circ} \mathrm{C}-\cdots$ - Cal. $f_{\gamma-\gamma^{\prime}}=0.01--$ Cal. $f_{\gamma-\gamma^{\prime}}=10.0$

Coarse -----Cal. $f_{\gamma-\gamma^{\prime}}=0.1-\cdot-\cdot-$ Cal. $f_{\gamma-y^{\gamma}}=100.0$

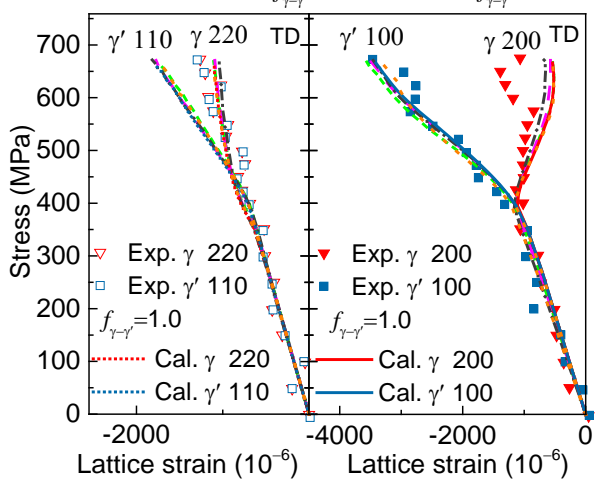

Fig. 9. Comparison among simulated lattice strains with different $f_{\gamma-\gamma^{\prime}}$ values for Alloy RR1000 with different $\gamma^{\prime}$ microstructures at $750{ }^{\circ} \mathrm{C}$ by employing 2-site model (co-rotation). Points are experimental data from Francis et al. [13. 


$$
\begin{aligned}
& -\gamma-\cdots-\cdots \gamma^{\prime}\left(f_{y-\gamma}=1.0\right) \\
& -\gamma^{-\cdots--} \gamma^{\prime}\left(f_{y-\gamma}=10.0\right) \\
& \gamma^{-\cdots--} \gamma^{\prime}\left(f_{\gamma-\gamma^{\prime}}=100.0\right)
\end{aligned}
$$

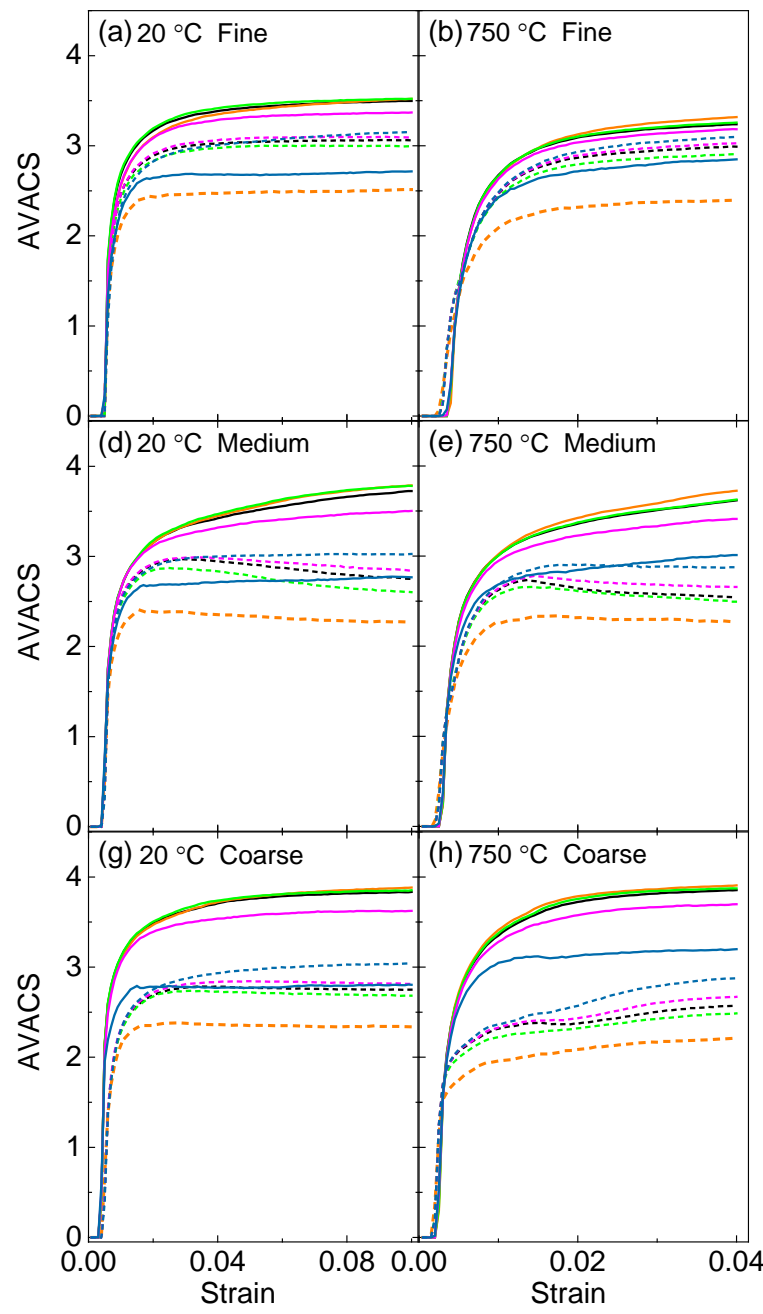

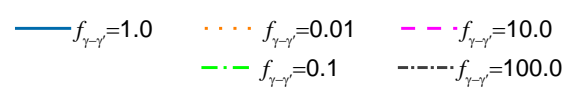

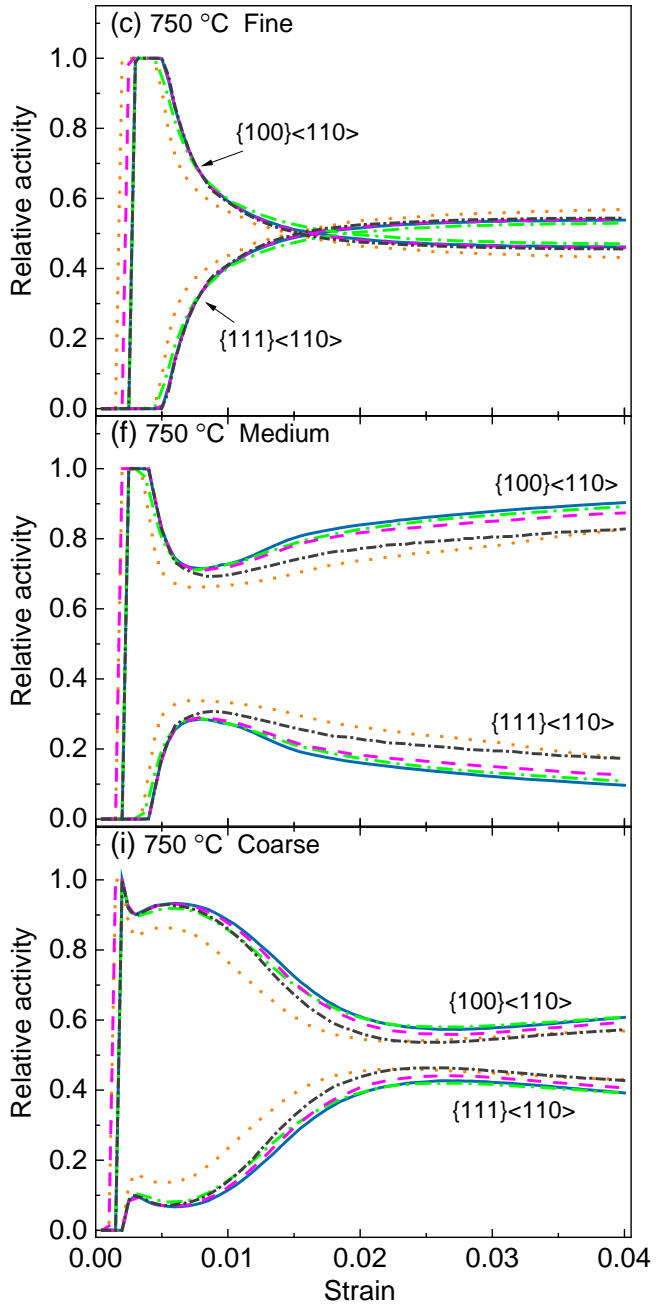

Fig. 10. Comparison among simulated AVACS at $20^{\circ} \mathrm{C}$ and $750{ }^{\circ} \mathrm{C}$ and relative activity of $\{111\}\langle 110\rangle$ and $\{100\}\langle 110\rangle$ slip systems in $\gamma^{\prime}$ phase at $750{ }^{\circ} \mathrm{C}$ with different $f_{\gamma-\gamma^{\prime}}$ values for Alloy RR1000 with different $\gamma^{\prime}$ microstructures by employing the 2 -site model (co-rotation). 


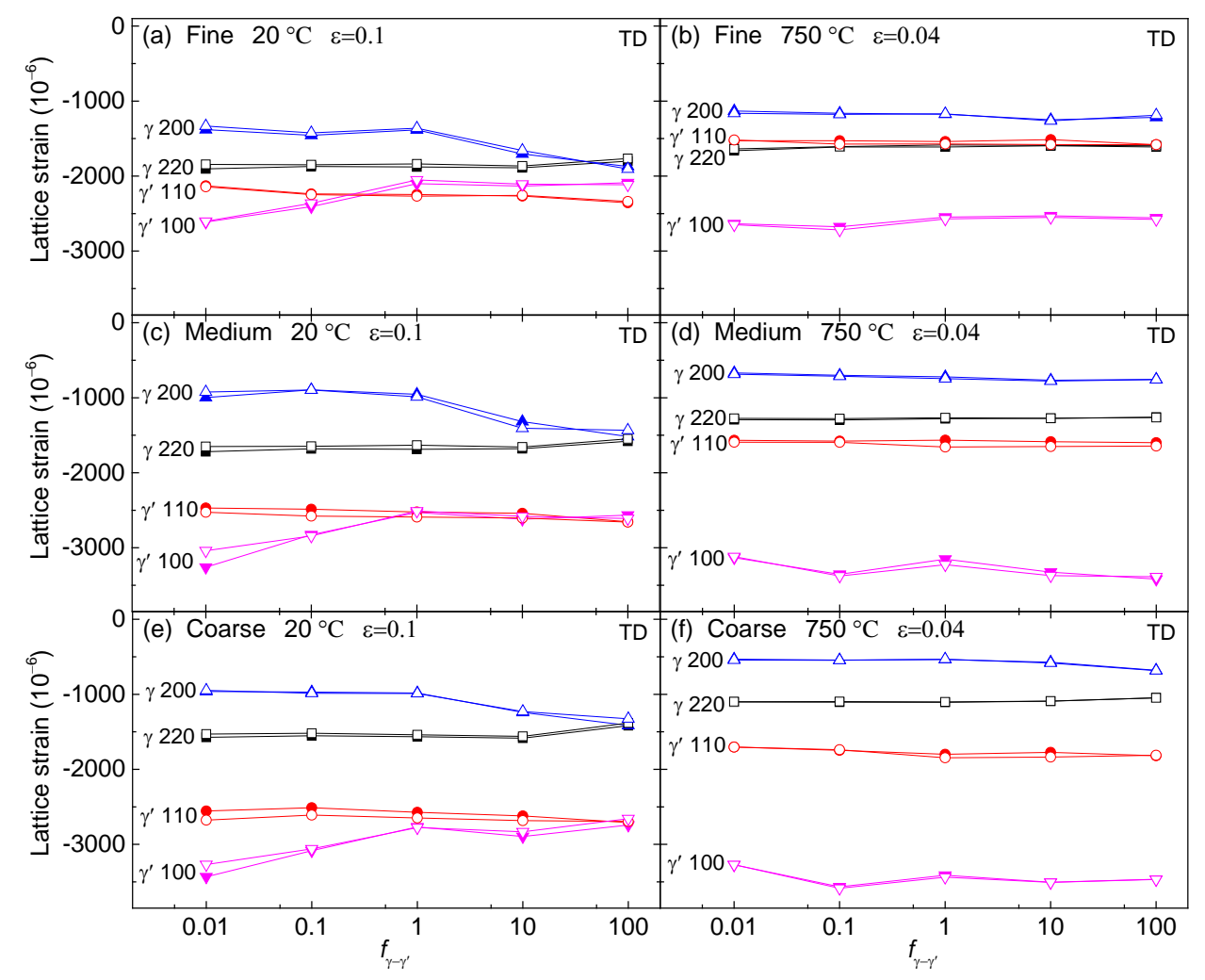

Fig. 11. Comparison among simulated transverse lattice strains with different $f_{\gamma-\gamma^{\prime}}$ values for Alloy RR1000 with different $\gamma^{\prime}$ microstructures at final deformation states of $\epsilon=0.10\left(20{ }^{\circ} \mathrm{C}\right)$ and $\epsilon=0.04\left(750{ }^{\circ} \mathrm{C}\right)$ by employing co-rotation (solid symbols) and local-co-rotation (hollow symbols) schemes in the 2-site model. Lines are used to guide the trend.

\section{Discussions}

\subsection{Grain-grain interaction}

Interaction between the two ellipsoids within each pair is the core of the 2-site EPSC model. Among all the physical quantities in the simulation, Ehshelby tensors are the most essential and critical, which are obtained from the Eshelby solution of the elastic equilibrium equation [19]. Moreover, the overall SC stiffness $\mathbf{L}$ is a function of symmetric Eshelby tensors (Eq. (10)), and the local rotation-rates (Eq. (12) ) used to update grain orientation distribution are in terms of both symmetric and anti-symmetric Eshelby tensors (Eq.(B.4) in Appendix B). A comprehensive understanding on correlation between the evolution of Eshelby tensors and the homogenized results is necessary, yet such knowledge is lacked from published work.

Results in Section 3.3 manifest that different $f_{\gamma-\gamma^{\prime}}$ values give various results of lattice strains for Alloy RR1000 with different $\gamma^{\prime}$ microstructures at both $20{ }^{\circ} \mathrm{C}$ and $750{ }^{\circ} \mathrm{C}$. In order to figure out the reason, we compared the coupling Eshelby tensors with different $f_{\gamma-\gamma^{\prime}}$ values at final deformation states of $\epsilon=0.10$ at $20{ }^{\circ} \mathrm{C}$ and $\epsilon=0.04$ at $750{ }^{\circ} \mathrm{C}$. Results of calculated 81 components of the coupling Eshelby tensors $\left(\mathbf{P}_{12}, \mathbf{P}_{21}, \boldsymbol{\Pi}_{12}\right.$, and $\left.\boldsymbol{\Pi}_{21}\right)$ are shown in Fig. 12 . The Eshelby tensors are weighted average values over all the ellipsoids in each phase. Results are similar for Alloy RR1000 with different $\gamma^{\prime}$ microstructures and therefore only the result of fine $\gamma^{\prime}$ microstructure is shown here to avoid redundancies. Panels (a-d) demonstrate that symmetric coupling Eshelby tensors of $\mathbf{P}_{12}$ increases and $\mathbf{P}_{21}$ decreases with increasing $f_{\gamma-\gamma^{\prime}}$, which means that $\gamma$ matrix suffers increased interaction from $\gamma^{\prime}$ precipitates, and vice 
versa. The same conclusion can be drawn for the anti-symmetric coupling Eshelby tensors of $\boldsymbol{\Pi}_{12}$ and $\boldsymbol{\Pi}_{21}$, as shown in panels (e-h). By comparing panels (a) to (c), (b) to (d), (e) to $(\mathrm{g})$, and $(\mathrm{f})$ to $(\mathrm{h})$, respectively, temperature dependence of the evolution of the coupling Eshelby tensors is found, which reveals that the exact value of each component is related to the plastic deformation mechanisms.
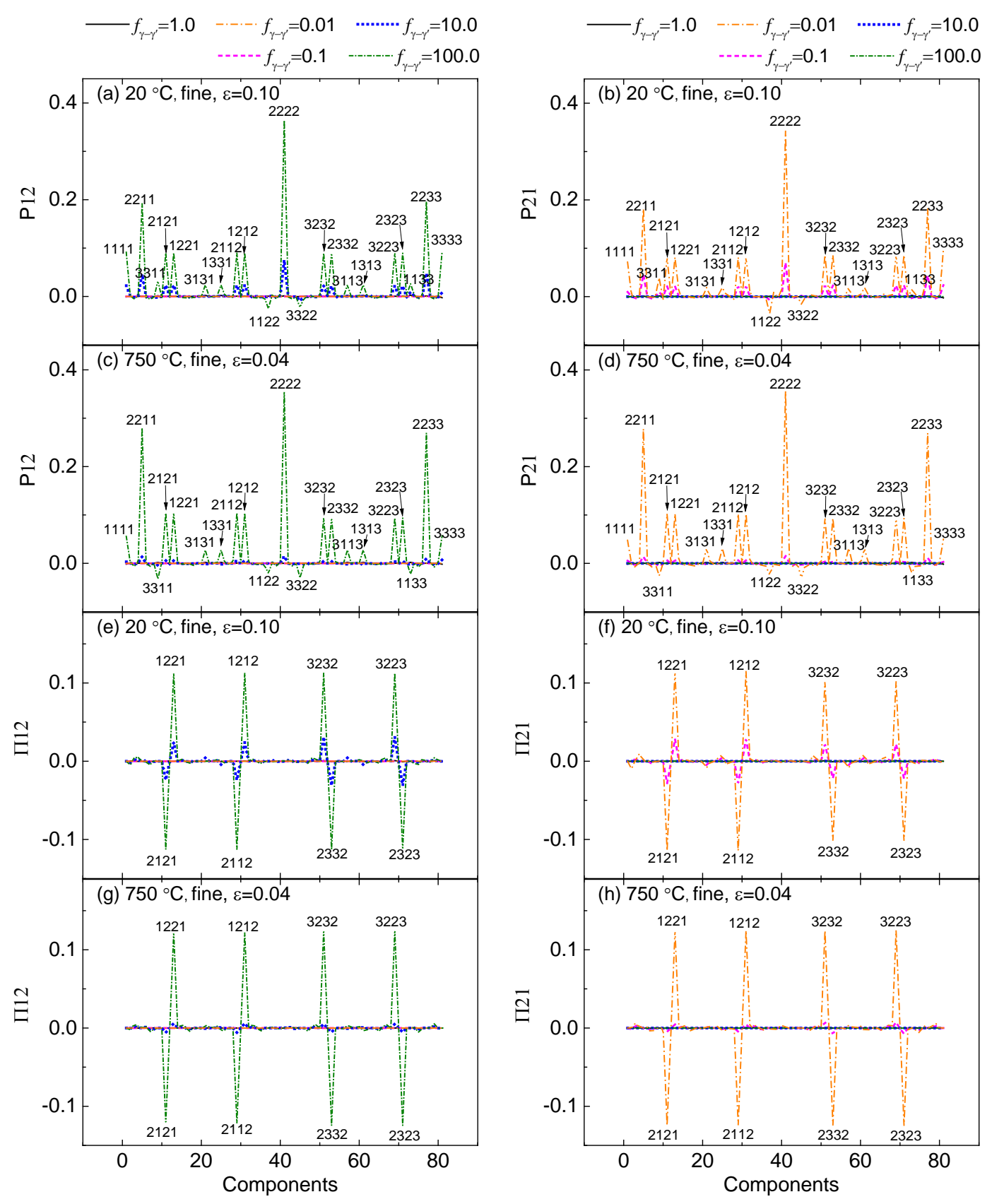

Fig. 12. Comparison between calculated 81 components of coupling Eshelby tensors $\left(\mathbf{P}_{12}, \mathbf{P}_{21}, \boldsymbol{\Pi}_{12}\right.$, and $\boldsymbol{\Pi}_{21}$ ) with different $f_{\gamma-\gamma^{\prime}}$ values for Alloy RR1000 with fine $\gamma^{\prime}$ microstructure at $20{ }^{\circ} \mathrm{C}$ and $750{ }^{\circ} \mathrm{C}$ studied in Section 3.3. The Eshelby tensors are weighted average values over all the grains in each phase. Some of the indices corresponding to their components are labelled. Panels (a-d) and (e-h) have the same coordinate range, respectively. 


\subsection{Re-fitted lattice strains}

From previous results in Sections 3.3 and 4.1 , we can find that $f_{\gamma-\gamma^{\prime}}$ has a significant effect on the homogenized results by changing $\gamma-\gamma^{\prime}$ interaction strength. In order to improve the predicted lattice strains for Alloy RR1000 with fine, medium, and coarse $\gamma^{\prime}$ microstructures at $20{ }^{\circ} \mathrm{C}$ and $750{ }^{\circ} \mathrm{C}$ studied in Section 3.1, we adjusted $f_{\gamma-\gamma^{\prime}}$ values for each case to perform re-simulation. By an optimization on $f_{\gamma-\gamma^{\prime}}$ value within the range of 0.1 to 10.0 for each case, a value around $f_{\gamma-\gamma^{\prime}}=8.0$ is found to distribute a best fit for all the cases at $20{ }^{\circ} \mathrm{C}$, but the results at $750{ }^{\circ} \mathrm{C}$ are not changed or improved much. In Grant et al. [12], it is also discovered that large number of inclusions (5000 50,000 pairs) is required to well capture the evolution of transverse lattice strains, and hence we increase the total number to 40,000 pairs to further improve the calculated transverse lattice strains. Re-calculated results are shown in Figs. 13 and 14. Since the axial lattice strains are not changed by using the increased $f_{\gamma-\gamma^{\prime}}$ and total grain number for all the cases at both temperatures, only transverse lattice strains are displayed. Previous simulated results of $f_{\gamma-\gamma^{\prime}}=1.0$ with 5000 pairs by utilizing the 2 -site model (co-rotation) are also plotted in the figures for each case as a comparison. Transverse lattice strains at the plastic deformation stage of selected cases (the best fit) marked by orange dashed rectangular boxes in the legends, labelled by orange solid rectangular boxes in panels (a-c) in the figures, are magnified and shown to the right of each panel, named as $\left(a^{\prime}\right),\left(b^{\prime}\right)$, and $\left(c^{\prime}\right)$, respectively, where the simulated results with 2-site model (non-rotation) and 1-site model (co-rotation) are also shown as a comparison.

Fig. 13(a-c) shows that transverse lattice strains of $\gamma(200)$ at the plastic deformation stage at $20{ }^{\circ} \mathrm{C}$ are improved by increasing $f_{\gamma-\gamma^{\prime}}$ from 1.0 to 8.0, indicating that the $\gamma$ matrix suffers larger interaction from the $\gamma^{\prime}$ precipitates than it puts on them. The re-predicted transverse lattice strain of $\gamma(200)$ of the fine $\gamma^{\prime}$ microstructure fits the experimental data well (Fig. 13 $\left(a^{\prime}\right)$ ), that of the medium $\gamma^{\prime}$ microstructure is also acceptable (Fig. 13 $\left(b^{\prime}\right)$ ), while that of the coarse $\gamma^{\prime}$ microstructure still has a large deviation from the experiment (Fig. $13\left(c^{\prime}\right)$ ). This is presumably due to the transition from particle shearing to Orowan looping when the precipitates exceed a critical size, which requires a more complicated description of the hardening behavior [12]. A possible way to account for the localized hardening is to introduce a thin reinforcement layer between $\gamma^{\prime}$ inclusions and the HEM, as Zecevic et al. did [21], but it is beyond the scope of present work. Panels (a-c) in Figs. 13 and 14 indicate that total grain number has slight influences on transverse lattice strains of $\gamma(220)$ and $\gamma^{\prime}(110)$ at $20{ }^{\circ} \mathrm{C}$ but great effects on that at $750{ }^{\circ} \mathrm{C}$. The simulated lattice strains of the coarse $\gamma^{\prime}$ microstructure at $750{ }^{\circ} \mathrm{C}$ are improved by increasing total grain number (Fig. 13(c)), but that of the fine and medium $\gamma^{\prime}$ microstructures are not as good as the previous.

By further, panels $\left(a^{\prime}-c^{\prime}\right)$ in Figs. 13 and 14 show that transverse lattice strains of $\gamma$ (200) and/or $\gamma^{\prime}$ (100) predicted by the 1-site model (co-rotation) have larger deviations from the experiment comparing to that predicted by the 2-site model (co-rotation), which is more obvious at $20^{\circ} \mathrm{C}$. It indicates that incorporating interaction between $\gamma$ matrix and $\gamma^{\prime}$ precipitates in the SC model is necessary to well capture the evolution of lattice strains. We can also find that predicted results with crystal lattice rotation (both 2-site and 1-site models) are more approaching the experimental data than that without it (2-site model, non-rotation scheme), especially for the lattice strains of $\gamma(200)$ and $\gamma^{\prime}(100)$ when $\epsilon>0.02$ or so. Therefore, not only is the $\gamma-\gamma^{\prime}$ interaction important in accurately capturing transverse lattice strains, but also the rotations of ellipsoid (Eq.(15)) and crystal lattice (Eq.(17)) are predominant after some accumulations of plastic deformation. 
(a) $20^{\circ} \mathrm{C}-\ldots$ - Cal. $f_{\gamma y \gamma}=8.0--$ Cal. $f_{\gamma \gamma y}=8.0(40,000$ pairs $)$

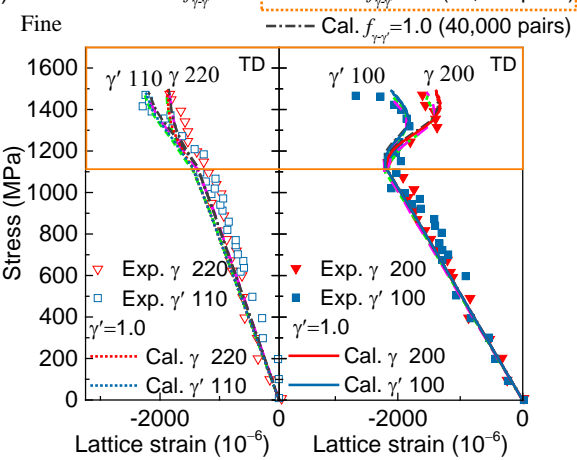

(b) $20{ }^{\circ} \mathrm{C}----$ Cal. $f_{1}=8.0--{ }^{2}$ Cal. $f=8.0(40,000$ pairs $)$ Medium $\quad-\cdot-\cdot-$ Cal. $f_{\gamma \gamma \gamma}=1.0$ (40,000 pairs)

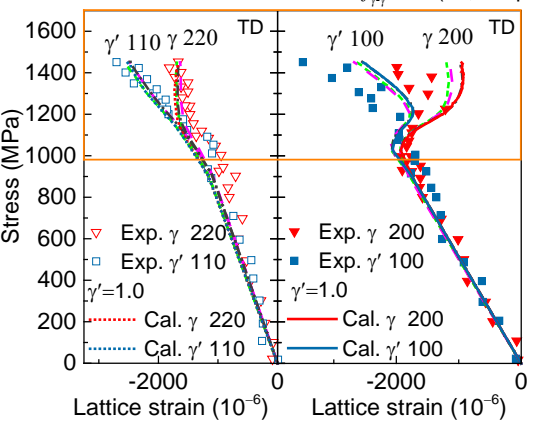

(c) $20^{\circ} \mathrm{C}---$ - Cal. $f_{\gamma \gamma}=8.0--$ Cal. $f_{y \gamma}=8.0(40,000$ pairs $)$ Coarse --.-- Cal. $f_{\gamma \gamma \gamma}=1.0$ (40,000 pairs)

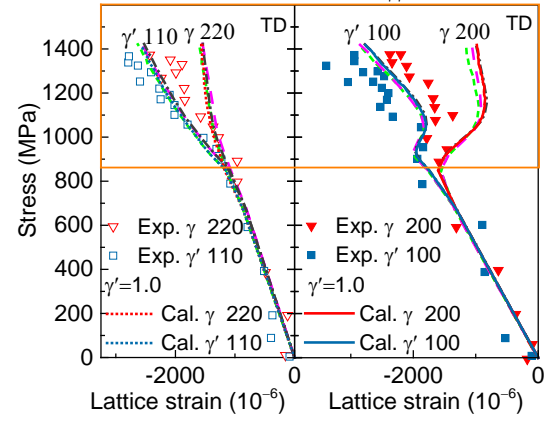

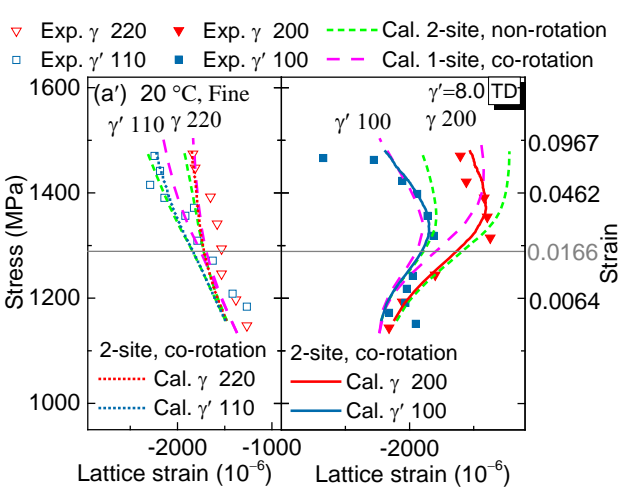

$\nabla$ Exp. $\gamma 220$ Vxp. $\gamma 200=---$ Cal. 2-site, non-rotation 口 Exp. $\gamma^{\prime} 110$ - Exp. $\gamma^{\prime} 100$ - - Cal. 1-site, co-rotation
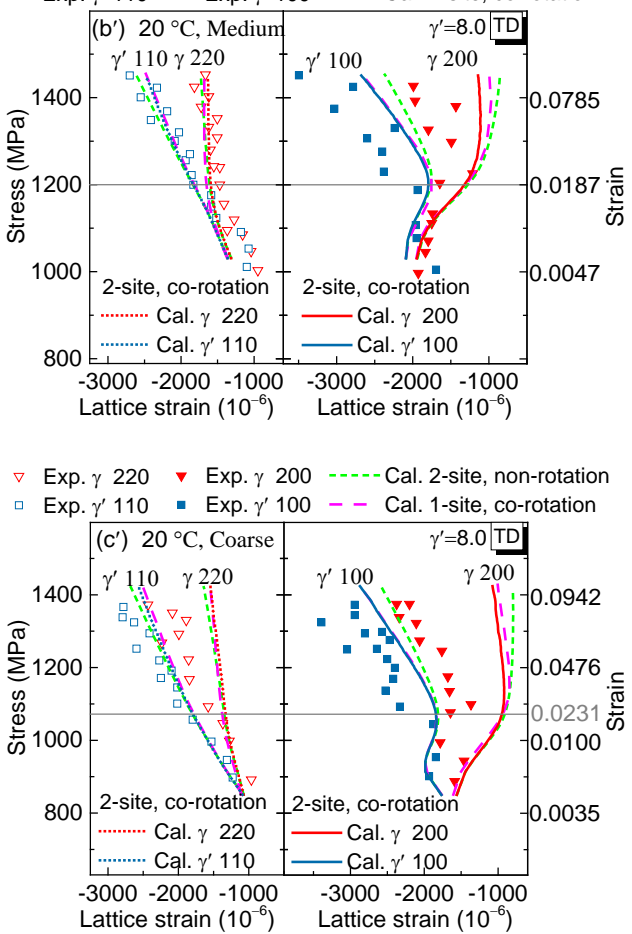

Fig. 13. Re-simulated lattice strains at $20^{\circ} \mathrm{C}$ by increasing $f_{\gamma-\gamma^{\prime}}$ and total grain number for Alloy RR1000 with (a) fine, (b) medium, and (c) coarse $\gamma^{\prime}$ microstructures, respectively. Previous simulated results of $f_{\gamma-\gamma^{\prime}}=1.0$ with 5000 pairs by employing the 2 -site model (co-rotation) are also shown as a comparison. Transverse lattice strains at the plastic deformation stage of selected cases marked by orange dashed rectangular boxes in the legends, labelled by orange solid rectangular boxes in panels (a-c), are magnified and shown to the right of each panel, named as $\left(a^{\prime}\right),\left(b^{\prime}\right)$, and $\left(c^{\prime}\right)$, respectively, where the simulated results with 2-site model (non-rotation) and 1-site model (co-rotation) are also shown as a comparison. Points are experimental data from Grant et al. 12. 

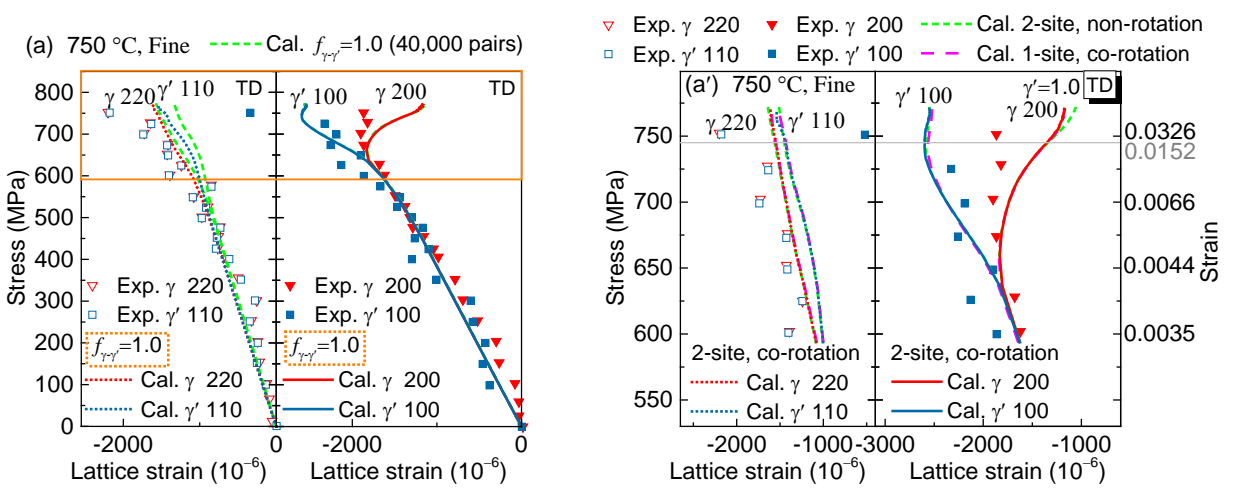

(b) $750^{\circ} \mathrm{C}$, Medium ---- - Cal. $f_{\gamma-\gamma}=1.0$ (40,000 pairs)
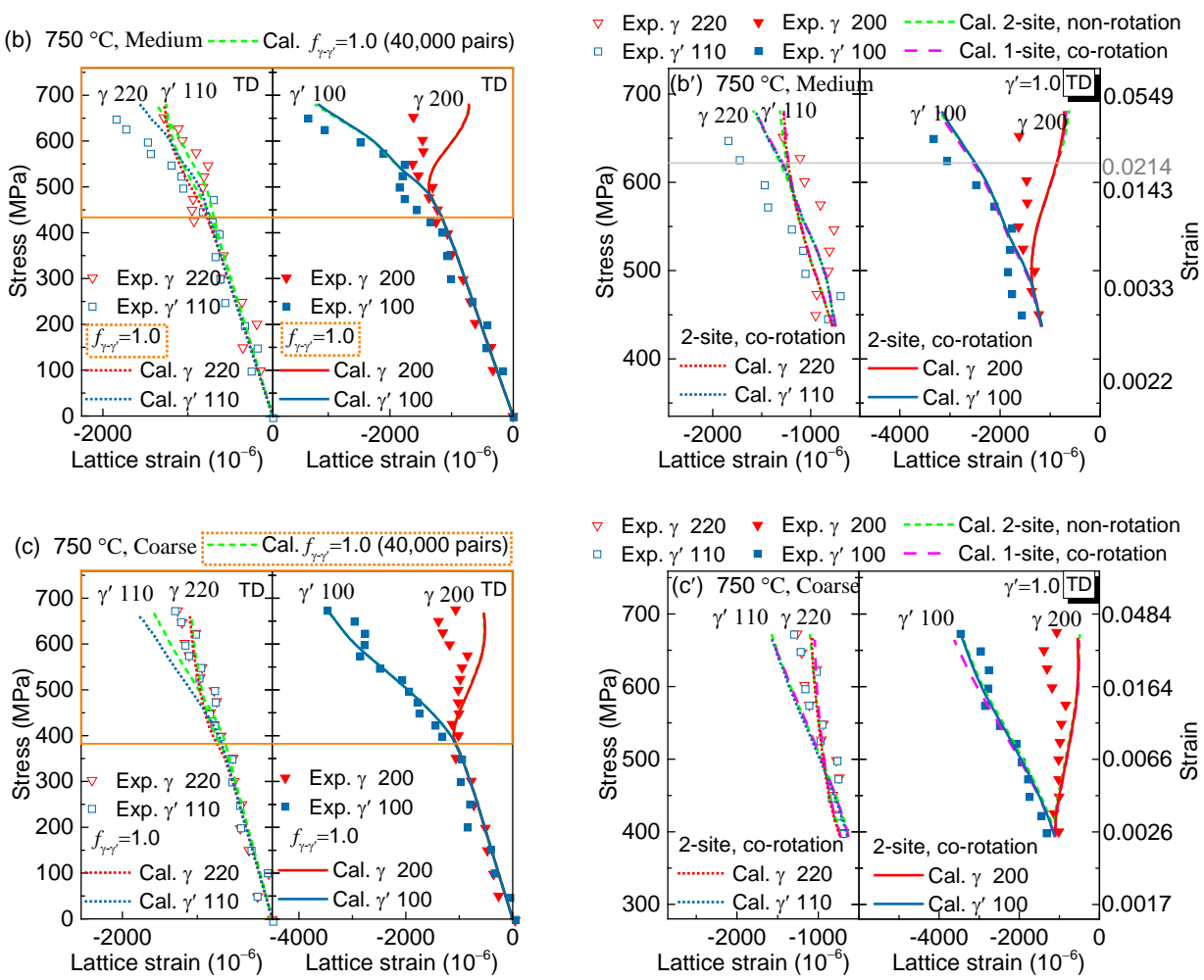

Fig. 14. Re-simulated lattice strains at $750{ }^{\circ} \mathrm{C}$ by increasing total grain number for Alloy RR1000 with (a) fine, (b) medium, and (c) coarse $\gamma^{\prime}$ microstructures, respectively. Previous simulated results of $f_{\gamma-\gamma^{\prime}}=1.0$ with 5000 pairs by employing the 2 -site model (co-rotation) are also shown as a comparison. Transverse lattice strains at the plastic deformation stage of selected cases marked by orange dashed rectangular boxes in the legends, labelled by orange solid rectangular boxes in panels (a-c), are magnified and shown to the right of each panel, named as $\left(a^{\prime}\right),\left(b^{\prime}\right)$, and $\left(c^{\prime}\right)$, respectively, where the simulated results with 2 -site model (non-rotation) and 1-site model (co-rotation) are also shown as a comparison. Points are experimental data from Francis et al. [13].

\subsection{Main factors affecting transverse lattice strains}

Diffraction technique gives the elastic strain averaged over all of the grain orientations that contribute to a given $h k l$ reflection, where subsets of grains within the defined gauge volume are different for AD and TD. In particular, the grains detected in AD have a similar orientation relative to the stress axis, while those in TD have a larger range of crystallographic orientation distribution compared with that of $\mathrm{AD}$ [24, 40]. It is found by Grant et al. 12 that 5000 and 50,000 pairs are required to well capture the evolution of transverse 
lattice strains of (100) and (110) reflections, respectively, while 1000 pairs are sufficient to model the axial.

In order to estimate the effect of grain number that contributes to each $h k l$ reflection on the predicted transverse lattice strains, we compared the grain number evolution for different reflections parallel and perpendicular to the tensile stress axis before and after refitting by employing the 2-site model (co-rotation), including previously used $f_{\gamma-\gamma^{\prime}}=1.0$ with 5000 pairs, increased $f_{\gamma-\gamma^{\prime}}=8.0$ with 5000 pairs, and $f_{\gamma-\gamma^{\prime}}=1.0$ with increased pairs of 40,000 at $20{ }^{\circ} \mathrm{C}$ and $750{ }^{\circ} \mathrm{C}$. Results are shown in Fig. 15 , where the vertical axis in each panel is the percentage relative to the total grain number of each phase. A contrast analysis between Fig. 13(a-c) and Fig. 15(a), (c), (e) indicates that the predicted transverse lattice strains of $\gamma(200)$ are improved by increasing the interaction factor $f_{\gamma-\gamma^{\prime}}$ to 8.0 while the corresponding "detected" grain number is decreased for all $\gamma^{\prime}$ microstructures at $20{ }^{\circ} \mathrm{C}$. Grain number percentage that contributes to $\gamma(220)$ and $\gamma^{\prime}(110)$ reflections along AD is largely increased by increasing total grain number to 40,000 pairs with that along TD is decreased at both temperatures, as shown in Fig. 15. The corresponding lattice strains of $\gamma(220)$ and $\gamma^{\prime}$ (110) along AD keep the previous values (not shown here) but that along TD are changed (panels (a-c) in Figs. 13 and 14). The above results demonstrate that grain orientation distribution plays a much more important role in the prediction of transverse lattice strains than the "detected" grain number does. This is consistent with Oliver et al.'s work [40], in which transverse lattice strain development with applied stress in cubic single crystals of specific orientations was studied by EPSC modeling. They found that transverse lattice strains show considerable variation depending upon the grain orientation with respect to the stress axis. Moreover, results shown in Section 4.2 reveal that transverse lattice strains of $\gamma$ (200) can be improved by changing $\gamma-\gamma^{\prime}$ interaction strength (represented by $\left.f_{\gamma-\gamma^{\prime}}\right)$ together with applying appropriate rotations to ellipsoid and crystal lattice. It is indicated that the interplay between interaction strength and evolution of grain orientation distribution is vital in the prediction of transverse lattice strains of $\gamma(200)$ and/or $\gamma^{\prime}(100)$. By contrast, the predicted transverse lattice strains of $\gamma(220)$ and $\gamma^{\prime}(110)$ are extremely sensitive to the initial crystallographic texture for the reason that they have more equivalent crystalline planes in a unit cell than the other reflections and they are more sensitive to any change of the initial grain orientation distribution. 

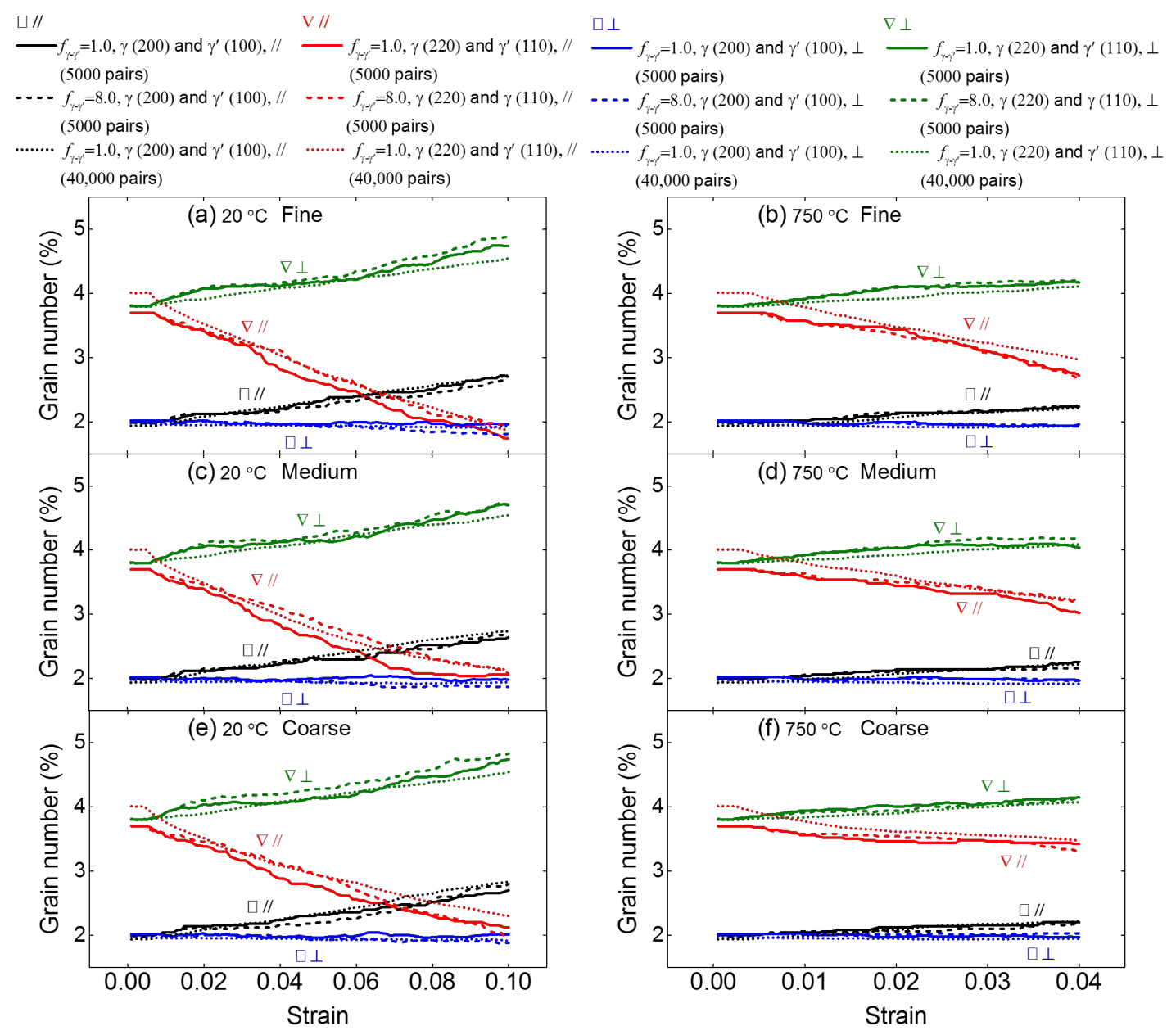

Fig. 15. Comparison of predicted grain number evolution for different reflections parallel and perpendicular to the tensile stress axis among previously used $f_{\gamma-\gamma^{\prime}}=1.0$ with 5000 pairs, increased $f_{\gamma-\gamma^{\prime}}=8.0$ with 5000 pairs, and $f_{\gamma-\gamma^{\prime}}=1.0$ with increased pairs of 40,000 at $20{ }^{\circ} \mathrm{C}$ and $750{ }^{\circ} \mathrm{C}$ by employing the 2 -site model (co-rotation). All the panels have the same vertical coordinate range. The symbols "//" and " $\perp$ " stand for reflection parallel and perpendicular to the tensile stress axis, respectively.

\section{Conclusions}

(1) A 2-site EPSC model is adapted for finite strain orientation-dependent two-phase case, and the predictive capability of the model is verified by adequately fitting published in situ neutron diffraction data for Ni-based superalloys at ambient and elevated temperatures.

(2) Predicted results are compared with 2-site model (small strain algorithm, non-rotation scheme) and 1-site model (finite strain algorithm, co-rotation scheme), which indicates that both crystal lattice rotation and grain-grain interaction are important for an accurate prediction of lattice strain evolution when the strain is larger than 0.02 for TD and $0.05 \sim 0.18$ for AD for the Ni-based superalloys studied in this work.

(3) Transverse lattice strains of $\gamma(200)$ and/or $\gamma^{\prime}$ (100) are sensitive to the interplay between $\gamma-\gamma^{\prime}$ interaction and evolution of grain orientation distribution with deformation, while that of $\gamma(220)$ and $\gamma^{\prime}$ (110) are sensitive to the initial crystallographic texture. 
(4) Experimental data of in situ neutron diffraction and texture at larger strains $(\epsilon \geqslant 0.15)$ is needed to verify and improve the model, and a detailed study on the development of rotation schemes is necessary to make the model more practical. Both are underway.

\section{Acknowledgements}

The work was funded by the Chalmers University of Technology Foundation through the project "Center for advanced neutron and x-ray scattering techniques" and Area of Advance Materials Science at Chalmers University of Technology. The work of Hongjia Li was also supported by the National Natural Science Foundation of China [Grant 51401193].

\section{Appendix A. The strain rates of an individual grain in both phases}

If thermal expansion is considered, the constitutive equations of a particular ellipsoidal inclusion (Eq. (19)) and the HEM (Eq.8) take the following form:

$$
\begin{aligned}
\dot{\boldsymbol{\sigma}}_{c} & =\mathbf{L}_{c}:\left(\dot{\boldsymbol{\epsilon}}_{c}-\alpha_{c} \dot{T}\right) \\
\dot{\boldsymbol{\sigma}} & =\mathbf{L}:(\dot{\boldsymbol{\epsilon}}-\alpha \dot{T})
\end{aligned}
$$

where $c=1,2$ for the two interacting inclusions in phase \# 1 and phase $\# 2 . \alpha_{c}, \alpha$, and $\dot{T}$ are the thermal expansion tensor of an individual inclusion, the overall thermal expansion tensor, and the temperature increment, respectively. The strain rates of an individual inclusion in phase \# 1 and \# 2 are given by:

$$
\begin{aligned}
& \dot{\boldsymbol{\epsilon}}_{1}=\mathbf{X}_{1}: \mathbf{Y}_{1}: \dot{\boldsymbol{\epsilon}}-\mathbf{X}_{1}: \mathbf{Z}_{1}: \mathbf{L}: \alpha \dot{T}+\mathbf{X}_{1}: \mathbf{W}_{1}: \mathbf{L}_{2}: \alpha_{2} \dot{T}+\mathbf{X}_{1}: \mathbf{V}_{1}^{-1}: \mathbf{L}_{1}: \alpha_{1} \dot{T} \\
& \dot{\boldsymbol{\epsilon}}_{2}=\mathbf{X}_{2}: \mathbf{Y}_{2}: \dot{\boldsymbol{\epsilon}}-\mathbf{X}_{2}: \mathbf{Z}_{2}: \mathbf{L}: \alpha \dot{T}+\mathbf{X}_{2}: \mathbf{W}_{2}: \mathbf{L}_{1}: \alpha_{1} \dot{T}+\mathbf{X}_{2}: \mathbf{V}_{2}^{-1}: \mathbf{L}_{2}: \alpha_{2} \dot{T}
\end{aligned}
$$

where the overall thermal coefficient $\alpha=\mathbf{L}^{-1}:\left\langle\mathbf{L}_{1}: \alpha_{1}+\mathbf{L}_{2}: \alpha_{2}\right\rangle$ and

$$
\begin{aligned}
\mathbf{X}_{1} & =\left(\mathbf{I}-\mathbf{V}_{1}^{-1}: \mathbf{S}_{1}: \mathbf{V}_{2}^{-1}: \mathbf{S}_{2}\right)^{-1} \\
\mathbf{Y}_{1} & =\mathbf{V}_{1}^{-1}:\left[\left(\mathbf{L}+\mathbf{Q}_{1}\right)+\mathbf{S}_{1}: \mathbf{V}_{2}^{-1}:\left(\mathbf{L}+\mathbf{Q}_{2}\right)\right] \\
\mathbf{Z}_{1} & =\mathbf{V}_{1}^{-1}:\left(\mathbf{S}_{1}: \mathbf{V}_{2}^{-1}+\mathbf{I}\right) \\
\mathbf{W}_{1} & =\mathbf{V}_{1}^{-1}: \mathbf{S}_{1}: \mathbf{V}_{2}^{-1}
\end{aligned}
$$

where $\mathbf{X}_{2}, \mathbf{Y}_{2}, \mathbf{Z}_{2}$, and $\mathbf{W}_{2}$ can be obtained interchanging indexes 1 and 2 in Eq.A.3. with

$$
\begin{aligned}
& \mathbf{V}_{1}=\mathbf{L}_{1}-\mathbf{L}:\left(\mathbf{I}-\mathbf{T}_{1}: \mathbf{U}_{1}\right) \\
& \mathbf{Q}_{1}=\mathbf{L}:\left(\mathbf{T}_{1}: \mathbf{U}_{1}-\mathbf{T}_{1}-\mathbf{I}\right) \\
& \mathbf{S}_{1}=\mathbf{L}: \mathbf{T}_{1}
\end{aligned}
$$

and that $\mathbf{V}_{2}, \mathbf{Q}_{2}$, and $\mathbf{S}_{2}$ can be obtained interchanging indexes 1 and 2 in Eq. (A.4).

The results above have been verified that $\mathbf{A}_{1}=\mathbf{X}_{1}: \mathbf{Y}_{1}$ and $\mathbf{A}_{2}=\mathbf{X}_{2}: \mathbf{Y}_{2}$ at $T=0$. 


\section{Appendix B. Deviations of local rotation-rates}

The calculation of the deviations of local rotation-rates from the average rotation-rate is similar to that for a two-site VPSC by Lebensohn and Canova [25].

Rewirte Eq. (3) as:

$$
\begin{aligned}
& \tilde{\dot{\boldsymbol{\epsilon}}}_{1}=\mathbf{P}_{11}: \dot{\boldsymbol{\epsilon}}_{1}^{*}+\mathbf{P}_{12}: \dot{\boldsymbol{\epsilon}}_{2}^{*} \\
& \tilde{\dot{\boldsymbol{\epsilon}}}_{2}=\mathbf{P}_{21}: \dot{\boldsymbol{\epsilon}}_{1}^{*}+\mathbf{P}_{22}: \dot{\boldsymbol{\epsilon}}_{2}^{*}
\end{aligned}
$$

Inverting Eq. B.1], we obtain

$$
\begin{aligned}
& \dot{\boldsymbol{\epsilon}}_{1}^{*}=\mathbf{T}_{1}: \mathbf{U}_{1}: \tilde{\dot{\boldsymbol{\epsilon}}}_{1}-\mathbf{T}_{1}: \tilde{\dot{\boldsymbol{\epsilon}}}_{2} \\
& \dot{\boldsymbol{\epsilon}}_{2}^{*}=-\mathbf{T}_{2}: \tilde{\dot{\boldsymbol{\epsilon}}}_{1}+\mathbf{T}_{2}: \mathbf{U}_{2}: \tilde{\dot{\boldsymbol{\epsilon}}}_{2}
\end{aligned}
$$

where:

$$
\begin{aligned}
& \mathbf{T}_{1}=\left(\mathbf{P}_{22}: \mathbf{P}_{12}{ }^{-1}: \mathbf{P}_{11}-\mathbf{P}_{21}\right)^{-1} \\
& \mathbf{U}_{1}=\mathbf{P}_{22}: \mathbf{P}_{12}{ }^{-1}
\end{aligned}
$$

and where $\mathbf{T}_{2}$ and $\mathbf{U}_{2}$ can be obtained interchanging indexes 1 and 2 in Eq.(B.3).

Deviations of the local strain rate from the average rotation-rates are calculated as:

$$
\begin{aligned}
& \tilde{\dot{\boldsymbol{\omega}}}_{1}=\mathbf{Y}_{11}: \tilde{\dot{\boldsymbol{\epsilon}}}_{1}+\mathbf{Y}_{12}: \tilde{\boldsymbol{\epsilon}}_{2} \\
& \tilde{\dot{\boldsymbol{\omega}}}_{2}=\mathbf{Y}_{21}: \tilde{\boldsymbol{\epsilon}}_{1}+\mathbf{Y}_{22}: \tilde{\boldsymbol{\epsilon}}_{2}
\end{aligned}
$$

where $\mathbf{Y}_{\alpha \beta}$ tensors are defined as:

$$
\begin{aligned}
& \mathbf{Y}_{11}=\boldsymbol{\Pi}_{11}: \mathbf{T}_{1}: \mathbf{U}_{1}-\boldsymbol{\Pi}_{12}: \mathbf{T}_{2} \\
& \mathbf{Y}_{12}=\boldsymbol{\Pi}_{12}: \mathbf{T}_{2}: \mathbf{U}_{2}-\boldsymbol{\Pi}_{11}: \mathbf{T}_{1}
\end{aligned}
$$

Here, $\boldsymbol{\Pi}_{\alpha \beta}$ are anti-symmetric Eshelby tensors, and $\mathbf{Y}_{22}$ and $\mathbf{Y}_{21}$ can be obtained by interchanging indexes 1 and 2 in Eq.(B.5). Related derivations and expressions for the two-site Eshelby tensors refer to Berveiller et al. [31] and Lebensohn and Canova [25].

\section{References}

[1] B. Clausen, T. Lorentzen, T. Leffers, Self-consistent modelling of the plastic deformation of f.c.c. polycrystals and its implications for diffraction measurements of internal stresses, Acta Materialia 46 (9) (1998) 3087 - 3098.

[2] M. R. Daymond, C. N. Tomé, M. A. M. Bourke, Measured and predicted intergranular strains in textured austenitic steel, Acta Materialia 48 (2) (2000) 553 - 564.

[3] S. R. Agnew, C. N. Tomé, D. W. Brown, T. M. Holden, S. C. Vogel, Study of slip mechanisms in a magnesium alloy by neutron diffraction and modeling, Scripta Materialia 48 (8) (2003) $1003-1008$.

[4] M. Bourke, S. Rajagopalan, R. Vaidyanathan, A. Little, Elastic modulus of shapememory niti from in situ neutron diffraction during macroscopic loading, instrumented indentation, and extensometry, Applied Physics Letters 86 (2005) 081901-081901-3. 
[5] S. R. Agnew, D. W. Brown, C. N. Tomé, Validating a polycrystal model for the elastoplastic response of magnesium alloy az31 using in situ neutron diffraction, Acta Materialia 54 (18) (2006) $4841-4852$.

[6] S. R. Agnew, R. P. Mulay, F. J. Polesak, C. A. Calhoun, J. J. Bhattacharyya, B. Clausen, In situ neutron diffraction and polycrystal plasticity modeling of a mg-y-nd-zr alloy: Effects of precipitation on individual deformation mechanisms, Acta Materialia 61 (10) (2013) $3769-3780$.

[7] D. W. Brown, B. Clausen, T. A. Sisneros, L. Balogh, I. J. Beyerlein, In situ neutron diffraction measurements during annealing of deformed beryllium with differing initial textures, Metallurgical and Materials Transactions A 44 (2013) 5665-5675.

[8] H. Wang, L. Capolungo, B. Clausen, C. N. Tomé, A crystal plasticity model based on transition state theory, International Journal of Plasticity 93 (2017) $251-268$.

[9] Y. Tomota, H. Tokuda, Y. Adachi, M. Wakita, N. Minakawa, A. Moriai, Y. Morii, Tensile behavior of trip-aided multi-phase steels studied by in situ neutron diffraction, Acta Materialia 52 (20) (2004) 5737 - 5745.

[10] M. R. Daymond, M. Preuss, B. Clausen, Evidence of variation in slip mode in a polycrystalline nickel-base superalloy with change in temperature from neutron diffraction strain measurements, Acta Materialia 55 (9) (2007) 3089 - 3102.

[11] O. Muransky, P. Sittner, J. Zrnik, E. C. Oliver, In situ neutron diffraction investigation of the collaborative deformation-transformation mechanism in trip-assisted steels at room and elevated temperatures, Acta Materialia 56 (14) (2008) 3367 - 3379.

[12] B. M. B. Grant, E. M. Francis, J. Q. da Fonseca, M. R. Daymond, M. Preuss, Deformation behaviour of an advanced nickel-based superalloy studied by neutron diffraction and electron microscopy, Acta Materialia 60 (19) (2012) 6829 - 6841.

[13] E. M. Francis, B. M. B. Grant, J. Q. da Fonseca, P. J. Phillips, M. J. Mills, M. R. Daymond, M. Preuss, High-temperature deformation mechanisms in a polycrystalline nickel-base superalloy studied by neutron diffraction and electron microscopy, Acta Materialia 74 (2014) $18-29$.

[14] V. Hounkpati, S. Fréour, D. Gloaguen, V. Legrand, J. Kelleher, W. Kockelmann, S. Kabra, In situ neutron measurements and modelling of the intergranular strains in the near- $\beta$ titanium alloy ti- $\beta 21$ s, Acta Materialia 109 (2016) $341-352$.

[15] D. W. Brown, M. A. M. Bourke, A. J. Clarke, R. D. Field, R. E. Hackenberg, W. L. Hults, D. J. Thoma, The effect of low-temperature aging on the microstructure and deformation of uranium- 6 wt \% niobium: An in-situ neutron diffraction study, Journal of Nuclear Materials 481 (2016) $164-175$.

[16] M. Zecevic, M. Knezevic, I. J. Beyerlein, C. N. Tomé, An elasto-plastic self-consistent model with hardening based on dislocation density, twinning and de-twinning: Application to strain path changes in hcp metals, Materials Science and Engineering: A 638 (2015) $262-274$. 
[17] R. Hill, Continuum micro-mechanics of elastoplastic polycrystals, Journal of the Mechanics and Physics of Solids 13 (2) (1965) 89 - 101.

[18] J. W. Hutchinson, Elastic-plastic behaviour of polycrystalline metals and composites, Proceedings of the Royal Society of London A: Mathematical, Physical and Engineering Sciences 319 (1537) (1970) 247-272.

[19] J. D. Eshelby, The determination of the elastic field of an ellipsoidal inclusion, and related problems, Proceedings of the Royal Society of London A: Mathematical, Physical and Engineering Sciences 241 (1226) (1957) 376-396.

[20] S. Berbenni, M. Cherkaoui, Homogenization of multicoated inclusion-reinforced linear elastic composites with eigenstrains: Application to thermoelastic behavior, Philosophical Magazine 90 (22) (2010) 3003-3026.

[21] M. Zecevic, Y. P. Korkolis, T. Kuwabara, M. Knezevic, Dual-phase steel sheets under cyclic tension-compression to large strains: Experiments and crystal plasticity modeling, Journal of the Mechanics and Physics of Solids 96 (2016) $65-87$.

[22] S. Ghorbanpour, M. Zecevic, A. Kumar, M. Jahedi, J. Bicknell, L. Jorgensen, I. J. Beyerlein, M. Knezevic, A crystal plasticity model incorporating the effects of precipitates in superalloys: Application to tensile, compressive, and cyclic deformation of inconel 718, International Journal of Plasticity 99 (2017) $162-185$.

[23] P. A. Turner, C. N. Tomé, A study of residual stresses in zircaloy-2 with rod texture, Acta Metallurgica et Materialia 42 (12) (1994) 4143 - 4153.

[24] C. J. Neil, J. A. Wollmershauser, B. Clausen, C. N. Tomé, S. R. Agnew, Modeling lattice strain evolution at finite strains and experimental verification for copper and stainless steel using in situ neutron diffraction, International Journal of Plasticity 26 (12) (2010) $1772-1791$.

[25] R. A. Lebensohn, G. R. Canova, A self-consistent approach for modelling texture development of two-phase polycrystals: Application to titanium alloys, Acta Materialia 45 (9) (1997) $3687-3694$.

[26] P. A. Turner, N. Christodoulou, C. N. Tomé, Modeling the mechanical response of rolled zircaloy-2, International Journal of Plasticity 11 (3) (1995) $251-265$.

[27] R. A. Lebensohn, C. N. Tomé, A self-consistent anisotropic approach for the simulation of plastic deformation and texture development of polycrystals: Application to zirconium alloys, Acta Metallurgica et Materialia 41 (9) (1993) 2611 - 2624.

[28] N. Jia, Z. Cong, X. Sun, S. Cheng, Z. Nie, Y. Ren, P. K. Liaw, Y. Wang, An in situ high-energy x-ray diffraction study of micromechanical behavior of multiple phases in advanced high-strength steels, Acta Materialia 57 (13) (2009) 3965 - 3977.

[29] C. N. Tomé, R. A. Lebensohn, C. T. Necker, Mechanical anisotropy and grain interaction in recrystallized aluminum, Metallurgical and Materials Transactions 33A (8) (2002) $2635-2648$. 
[30] T. Mura, Micromechanics of defects in solids, Springer, Dordrecht, 1987.

[31] M. Berveiller, O. Fassi-Fehri, A. Hihi, The problem of two plastic and heterogeneous inclusions in an anisotropic medium, International Journal of Engineering Science 25 (6) (1987) $691-709$.

[32] C. T. Sims, N. S. Stoloff, W. C. Hagel, Superalloys II: high-temperature materials for aerospace and industrial power, New York: John Wiley \& Sons, 1987.

[33] B. Clausen, C. N. Tomé, D. W. Brown, S. R. Agnew, Reorientation and stress relaxation due to twinning: Modeling and experimental characterization for mg, Acta Materialia 56 (11) (2008) $2456-2468$.

[34] U. F. Kocks, C. N. Tomé, H. R. Wenk, Texture and anisotropy: preferred orientation in polycrystals and their effect on materials properties, Cambridge University Press, 1998.

[35] C. N. Tomé, P. J. Maudlin, R. A. Lebensohn, G. C. Kaschner, Mechanical response of zirconium - i. derivation of a polycrystal constitutive law and finite element analysis, Acta Materialia 49 (15) (2001) 3085 - 3096.

[36] P. H. Thornton, R. G. Davies, T. L. Johnston, The temperature dependence of the flow stress of the $\gamma^{\prime}$ phase based upon ni3al, Metallurgical Transactions 1 (1) (1970) 207-218.

[37] A. E. Staton-Bevan, R. D. Rawlings, The deformation behaviour of single crystal ni3(al, ti), physica status solidi (a) 29 (2) (1975) 613-622.

[38] R. Hielscher, H. Schaeben, A novel pole figure inversion method: specification of the MTEX algorithm, Journal of Applied Crystallography 41 (6) (2008) 1024-1037.

[39] D. Mainprice, R. Hielscher, H. Schaeben, Calculating anisotropic physical properties from texture data using the mtex open-source package, Geological Society of London Special Publications 360 (2011) 175-192.

[40] E. C. Oliver, M. R. Daymond, P. J. Withers, Interphase and intergranular stress generation in carbon steels, Acta Materialia 52 (7) (2004) 1937 - 1951. 